Western University

Scholarship@Western

Biochemistry Publications

Biochemistry Department

4-1-2015

\title{
Molecular effects of cancer-associated somatic mutations on the structural and target recognition properties of Keapl.
}

Halema Khan

Ryan C Killoran

Anne Brickenden

Jingsong Fan

Daiwen Yang

See next page for additional authors

Follow this and additional works at: https://ir.lib.uwo.ca/biochempub

Part of the Biochemistry Commons

Citation of this paper:

Khan, Halema; Killoran, Ryan C; Brickenden, Anne; Fan, Jingsong; Yang, Daiwen; and Choy, Wing-Yiu, "Molecular effects of cancerassociated somatic mutations on the structural and target recognition properties of Keap1." (2015). Biochemistry Publications. 174.

https://ir.lib.uwo.ca/biochempub/174 
Authors

Halema Khan, Ryan C Killoran, Anne Brickenden, Jingsong Fan, Daiwen Yang, and Wing-Yiu Choy 


\title{
Molecular effects of cancer-associated somatic mutations on the structural and target recognition properties of Keap1
}

\author{
Halema Khan ${ }^{\dagger}$, Ryan C. Killoran ${ }^{\dagger}$, Anne Brickenden ${ }^{\dagger}$, Jingsong Fan ${ }^{\ddagger}$, Daiwen \\ Yang $^{\ddagger}$, and Wing-Yiu Choy ${ }^{\dagger *}$ \\ "Department of Biochemistry, The University of Western Ontario, London, Ontario, \\ Canada N6A 5C1. "Department of Biological Sciences, National University of Singapore, \\ 14 Science Drive 4, Singapore 117543 \\ *Corresponding author \\ E-mail: jchoy4@uwo.ca \\ Tel: 1-519-661-3161 \\ Fax: 1-519-661-3175
}

Short (page heading) title: Keap1 mutations 


\begin{abstract}
Kelch-like ECH-associated protein 1 (Keap1) plays an important regulatory role in the Nrf2-dependent oxidative stress response pathway. It functions as a repressor of Nuclear factor erythroid 2-related factor 2 (Nrf2), a key transcription factor that initiates the expression of cytoprotective enzymes during oxidative stress to protect cells from damage caused by reactive oxygen species. Recent studies show that mutations of Keap1 can lead to aberrant activation of the antioxidant pathway, which is associated with different types of cancers. To gain a mechanistic understanding of the links between Keap1 mutations and cancer pathogenesis, we have investigated the molecular effects of a series of mutations (G333C, G350S, G364C, G379D, R413L, R415G, A427V, G430C, and G476R) on the structural and target recognition properties of Keap1 by using nuclear magnetic resonance spectroscopy, circular dichroism, and isothermal titration calorimetry. Depending on their locations in the protein, these mutations are found to exert differential effects on the protein stability and target binding. Together with the proposed Hinge-and-Latch mechanism of Nrf2/Keap1 binding in literature, our results provide important insight into the molecular impact of different somatic mutations on Keap1's function as a Nrf2 repressor.
\end{abstract}

\title{
SUMMARY STATEMENT (40 words)
}

We have elucidated the molecular effects of nine cancer-associated somatic mutations of Keap1, an important regulator of the Nrf2-mediated oxidative stress response, on the structural integrity and target recognition properties of the protein by using multiple biophysical techniques. 
Keywords: nuclear magnetic resonance; isothermal titration calorimetry; oxidative stress response; Nrf2; Prothymosin $\alpha$

Abbreviations: BTB, Broad complex, Tramtrack and Bric-a-Brac; CD, circular dichroism; Cul 3, Cullin 3; HSQC, heteronuclear single quantum coherence; ITC, isothermal titration calorimetry; IVR, Intervening region; Keap1, Kelch-like ECHassociated protein 1; NMR, nuclear magnetic resonance; Nrf2, Nuclear factor erythroid 2-related factor 2; ProT $\alpha$, Prothymosin $\alpha$ 


\section{INTRODUCTION}

The Nrf2/Keap1 antioxidant pathway plays critical roles both in chemoprevention and cancer pathogenesis [1-4]. The transcription factor Nuclear factor erythroid 2-related factor-2 (Nrf2) induces the cellular defenses against reactive oxygen species and toxic substances by initiating the transcription of an array of cytoprotective genes, which are essential for the elimination of electrophilic and oxidative stresses before damage is caused to the cellular macromolecules $[5,6]$. Under normal conditions, its activity is suppressed by Keap1 (Kelch-like ECH-associated protein 1) in conjunction with the Cullin 3 (Cul3) E3 ubiquitin ligase complex via ubiquitin-mediated proteasomal degradation [5, 7-9]. In the presence of oxidative stress, however, specific cysteine residues in Keap1 are subjected to modifications by the oxidants [10]. The modified Keap1 is incapable of targeting Nrf2 for ubiquitination, allowing Nrf2 to translocate into the nucleus to initiate gene transcription [6].

Accumulation of Nrf2 in the nucleus however, can lead to aberrant cytoprotective gene expression if not regulated [11-13]. Keap1 is a cytosolic protein that lacks a nuclear localization signal and cannot enter the nucleus on its own to regulate Nrf2 levels [14]. Recent studies have revealed that the Keap1 protein can be shuttled to the nucleus in complex with Prothymosin $\alpha$ (ProT $\alpha$ ), an intrinsically disordered protein that contains a nuclear localization signal [14]. Once in the nucleus, Keap1 mediates ubiquitination and proteasomal degradation of Nrf2, causing Nrf2 to return to its basal level. This regulatory mechanism is essential as the continuous expression of cytoprotective proteins can promote cell proliferation leading to tumorigenesis $[1,15,16]$.

The Keap1 is comprised of five domains (Figure 1A), the N-terminal domain, the Broad complex, Tramtrack and Bric-a-Brac (BTB) domain, the intervening region (IVR), the Kelch domain, and the C-terminal region, and all these domains contribute to Nrf2 repression. The $\mathrm{N}$-terminal, BTB, IVR and the $\mathrm{C}$-terminal regions contain critical cysteine residues that are important for Keap1 activity [17-20]. The BTB domain is also responsible for Keap1 dimerization. This domain, together with the IVR domain, mediates the Keap1 interaction with the Cul3-E3 ligase complex required for the ubiquitination of $\mathrm{Nrf2}$ [7, 8, 21]. The Kelch domain is responsible for target recognition. Besides Nrf2 and ProT $\alpha$, the domain has also been shown to interact with WTX [22], p62 [23], PGAM5 [24], PALB2 [25], FAC1 [26], and IKK $\beta$ [27] to name a few [28]. In the case of Nrf2, the N-terminal Neh2 domain of the protein harbors two Kelch-binding motifs, the ETGE and DLG motifs, that interact with Keap1 through a "Hinge and Latch" mechanism: one Kelch domain of the Keap1 dimer recruits the Nrf2 by first forming a complex with the high binding affinity ETGE motif and then the second Kelch domain of the dimer captures the weaker DLG motif of the same Nrf2 protein [29,30]. This binding mechanism allows the lysine residues located in between these two motifs in the Neh2 domain to be properly positioned for ubiquitination $[9,29,30]$. On the other hand, other targets of Keap1 like ProT $\alpha$ form a complex with the Kelch domain in a one to one stoichiometry [31].

Over 200 somatic mutations within Keap1 have been reported in various types of cancer tissues and cancer-derived cell lines [12, 15, 16, 32-34]. Several of them have 
been shown to be defective in inhibiting Nrf2 activity $[15,16,35]$, resulting in constitutive expression of cytoprotective enzymes in cancer cells thus promoting cell survival [11]. It has been speculated that these mutations compromise Keapl's structural integrity, rendering it incapable of promoting the ubiquitination of $\mathrm{Nrf} 2[11,36]$. However, only a few studies of the structural and target-binding properties of these mutants have been carried out to date $[32,36]$. Therefore, the molecular links between these mutations and the diseases remain poorly understood.

To address this problem, we set out to elucidate the effects of disease-associating missense mutations on the structural integrity and target recognition of Keap1 by using nuclear magnetic resonance (NMR) spectroscopy, circular dichroism (CD) spectropolarimetry and isothermal titration calorimetry (ITC). We have chosen to focus on the Kelch domain (residue 321-609) of Keap1 in this study. Importantly, close to 50\% of the missense somatic mutations identified in Keap1 so far are found to reside in this domain (based on the data in the database of the Catalogue of Somatic Mutations in Cancer-COSMIC [37]). Crystallographic studies show that the Kelch domain adopts a six-bladed $\beta$-propeller conformation (Figure 1B) with several conserved amino acids that are important for maintaining the hydrogen bond network, linking the propeller blades and forming the hydrophobic core of the protein $[36,38,39]$. In particular, there is a conserved glycine doublet found in each blade of the Kelch domain. These two glycine residues are involved in an intrablade hydrogen bond network that may be instrumental in the folding of individual blade structures. Both the ETGE and DLG motifs of Nrf2, as well as ProT $\alpha$ are found to bind to the same positively charged pocket located at the mouth of the central tunnel of the Kelch $\beta$-propeller [36, 40, 41]. The binding interface of Kelch with targets is demonstrated in the crystal structure of Neh2-ETGE peptide bound to the Kelch domain shown in Figure 1B [36].

We have investigated the effects of nine cancer-associated somatic mutations [11], G333C, G350S, G364C, G379D, R413L, R415G, A427V, G430C, G476R, on the target recognition and structural integrity of the Kelch domain of Keap1 (Table S1) [11, $12,15,16,33,35,38]$. These mutants can be divided into three categories based on their location in the Kelch domain. The R415 residue is one of the conserved Arg residues located in the positively charged vicinity in the bottom face of the propeller (Figure 1C) and it is directly involved in the interface formation with ProT $\alpha$ and Nrf2 [36, 38, 40]. The G364C and R413L mutants are also included in the same category as the R415G. Even though these two residues do not directly participate in target binding, they are both next to residues that are involved in the ProT $\alpha$ and Nrf2 interactions (Figure 1C) [36, 38, 40]. Conversely, the second set of mutations, G350S and A427V, are distant from the binding interface. The G350S as shown in Figure 1D is at the opposite side of the basic pocket, and the $\mathrm{A} 427 \mathrm{~V}$ is found within a $\beta$-strand of blade III of the propeller. The last group, containing G333C, G379D, G430C, and G476R, are mutations that occur in the conserved double glycine repeats located in different blades (Figure 1E) [39]. Three of these four mutations, G333C, G379D and G430C, occur in the second position of the GG repeat while the G476R occurs in the first position of the doublet. Since these glycine residues are expected to play an important structural role in the Kelch domain, it is 
important to assess the impacts of these mutations on the domain's structural integrity and function.

NMR and ITC were used to examine the interactions between Nrf2/ProT $\alpha$ and the Kelch mutants. Nrf2 and ProT $\alpha$ have been shown to have the strongest and the weakest binding affinity, respectively, to the wild-type Kelch domain among many of the known partners studied [42]. Additionally, we have completed the backbone NMR chemical shift assignment of the $34 \mathrm{kDa}$ Kelch domain of human Keap1 [43]. This affords an effective way to probe the conformational changes induced upon mutating specific residues in the Kelch domain and provides a manner to observe regions important for target binding. Our results show that these somatic mutations exert differential effects on the structural integrity and target recognition properties of Keap1, implying that their molecular links to the cancer pathogenesis can be very distinct. 


\section{MATERIALS AND METHODS}

\section{Cloning, expression and purification of Keap1-Kelch variants}

Mutant constructs of the Kelch domain were generated using the QuikChange II site-directed mutagenesis kit (Stratagene) with the wild-type human Kelch domain (residues 321-609 of Keap1) in the prokaryote expression vector pDEST17 (Life Technologies) [39]. Following are the primers used for the mutations: G333C-5'ctacaccgegggetgctacttccgaca-3'; G350S-5'-caaccccagtgacagcacctggctccg-3'; G364C-5'aggtgccgcggagctgcetggceggctgcg-3'; G379D-5'-gtacgccgtgggegacaggaacaactcgcc-3'; R413L-5'-atgagcgtgccctttaaccgcatcggg-3'; R415G-5'-agcgtgccccgtaacggcatcggggtgg-3'; A427V-5'-gatggccacatctatgtcgtcggcggct-3'; G430C-5'-atatgccgtcggctgctcccacggctg-3'; G476R-5'-tgctttatgccgtgaggggctttgacggg-3'. Wild-type and mutational variants (G333C, G350S, G364C, G379D, R413L, R415G, A427V, G430C, G476R) were expressed and purified following the protocols described in [31].

\section{Neh2 constructs, expression and purification}

The gene fragment of human Neh2 domain of Nrf2 (amino acid residues M1G98) was cloned out from the full length Nrf2 (purchased from Invitrogen; NM_006164) and then inserted into the Gateway pDEST17 vector. The Neh2 $\triangle E T G E$ mutant (deletion of ${ }^{79} \mathrm{ETGE}^{82}$ from Neh2) was constructed from the full-length Neh2 fragment using the QuikChange II site-directed mutagenesis kit (Stratagene). For each construct, a Tobacco Etch virus (TEV) cleavage recognition site, ENLYFQG, was inserted between the histidine tag and the Neh2 fragment. Three constructs were transformed into the Rosetta 2 (DE3)pLysS (Novagen) strain of Escherichia coli (E. coli) for protein expression in M9 media. Cells were grown at $37^{\circ} \mathrm{C}$ and protein over-expression was induced at an $\mathrm{OD}_{600}$ of 0.6 with $0.5 \mathrm{mM}$ of isopropyl $\beta$-D-thiogalactopyranoside (IPTG, BioShop). The cells were allowed to grow overnight at $25^{\circ} \mathrm{C}$ before harvesting. The Neh2 protein and its variants were purified batch wise by Nickel-Sepharose (GE Healthcare) affinity chromatography, followed by his-tag removal using TEV protease at $25^{\circ} \mathrm{C}$ for 21 hours. Uncleaved Neh2 and the his-tagged TEV protease were then removed by NickelSepharose affinity chromatography. The final protein sample was analyzed on SDSPAGE for purity and dialyzed into $50 \mathrm{mM}$ sodium phosphate buffer, $100 \mathrm{mM} \mathrm{NaCl}, 1$ mM DTT, $\mathrm{pH} 7.0$ for NMR and ITC experiments.

\section{Peptides}

The ProT $\alpha$-ENGE peptide ( $>98 \%$ purity), ${ }^{33}$ APANGNANEENGEQEADNEV ${ }^{52}$, and the Neh2-ETGE peptide (>98\% purity), ${ }^{69}$ AFFAQLQLDEETGEFL ${ }^{84}$, were ordered from GenScript USA Inc. The Neh2-DLG peptide, ${ }^{20}$ IDILWRQDIDLGVSREVFDF ${ }^{39}$, was ordered from the Tufts University Core Facility with a purity level above $95 \%$. The lyophilized peptides were dissolved and dialyzed into $50 \mathrm{mM}$ sodium phosphate buffer, $100 \mathrm{mM} \mathrm{NaCl}$, and $1 \mathrm{mM}$ DTT for ITC and NMR experiments.

\section{Nuclear magnetic resonance (NMR) experiments}

For the backbone resonance assignment of the Kelch domain of human Keap1, NMR experiments were performed at $25^{\circ} \mathrm{C}$ on a Bruker Avance $800 \mathrm{MHz}$ (National University of Singapore) spectrometer equipped with a cryogenic probe. A $600 \mu \mathrm{M}^{2} \mathrm{H}-$ ${ }^{15} \mathrm{~N}-{ }^{13} \mathrm{C}$ labeled sample of the Kelch domain was used to acquire ${ }^{1} \mathrm{H}-{ }^{15} \mathrm{~N}-\mathrm{HSQC}$, 
$\mathrm{HN}(\mathrm{CO}) \mathrm{CACB}, \mathrm{HNCACB}$, and ${ }^{15} \mathrm{~N}-\mathrm{NOESY}-\mathrm{HSQC}$ spectra for sequential assignment of the protein. The data was processed with NMRPipe [44] and analyzed using CARA (http://cara.nmr.ch). The chemical shifts were deposited in the Biological Magnetic Resonance Bank (accession number 19992).

${ }^{15} \mathrm{~N}$-labeled samples of the Kelch domain were prepared at a $200 \mu \mathrm{M}$ concentration for the ligand-binding NMR experiments. ${ }^{1} \mathrm{H}-{ }^{15} \mathrm{~N}$ HSQC experiments were performed on a Varian INOVA $600 \mathrm{MHz}$ spectrometer with a cryogenic probe (UWO Biomolecular NMR facility) at $25^{\circ} \mathrm{C}$. Data was analyzed using NMRPipe [44] and NMRView [45].

\section{Circular Dichroism (CD) Spectropolarimetry}

Wild-type and Kelch mutant protein solutions $(\sim 2.2 \mathrm{mg} / \mathrm{mL})$ were each prepared in $50 \mathrm{mM}$ sodium phosphate buffer, $100 \mathrm{mM} \mathrm{NaCl}, 1 \mathrm{mM}$ DTT, pH 7.0. Far-UV CD spectra were obtained in a $0.1 \mathrm{~mm}$ cuvette using a Jasco J-810 spectropolarimeter (Easton, MD), at $25^{\circ} \mathrm{C}$ for wavelengths ranging from $190-260 \mathrm{~nm}$. The final curves for each sample represent the mean of twenty separate scans. The CD data $(\theta$, in millidegrees) were converted to mean residue ellipticity $([\theta])$ using standard formula and the software supplied by the manufacturer. The programs SELCON3, CDSSTR, and CONTIN provided by the DichroWeb were used to deconvolute the CD spectra [46].

\section{Isothermal Titration Calorimetry}

ITC experiments were carried out on a MicroCal ${ }^{\mathrm{TM}}$ VP-ITC instrument. All purified protein samples were dialyzed into $50 \mathrm{mM}$ phosphate buffer with $100 \mathrm{mM} \mathrm{NaCl}$ and $1 \mathrm{mM}$ DTT at $\mathrm{pH}$ 7.0. In typical experiments, wild-type Kelch and mutational variants were prepared to a concentration of $\sim 15 \mu \mathrm{M}$ for the Neh2-ETGE peptide $(\sim 150$ $\mu \mathrm{M})$ titrations and $\sim 60 \mu \mathrm{M}$ for the ProT $\alpha$ peptide $(\sim 800 \mu \mathrm{M})$ and Neh2-DLG peptide $(\sim 1$ $1.5 \mathrm{mM}$ ) titrations. Kelch or its mutational variants were loaded into the $1.42-\mathrm{mL}$ cell, and ligands were loaded into the syringe $(300 \mu \mathrm{L})$. For the full-length Neh2 and Neh2 $\Delta$ ETGE experiments with the wild-type and mutants of Kelch, the Kelch was loaded into the syringe and the Neh2 proteins were loaded into the cell. Titrations were performed at $25{ }^{\circ} \mathrm{C}$ (unless specified otherwise) starting with initial injection of $3 \mu \mathrm{L}$, followed by 59 injections of $5 \mu \mathrm{L}$, with spacing of 240 seconds to 300 seconds. For the Neh2 $\triangle$ ETGE construct, titrations were performed at $25{ }^{\circ} \mathrm{C}, 20^{\circ} \mathrm{C}, 15^{\circ} \mathrm{C}, 10{ }^{\circ} \mathrm{C}$ and $5{ }^{\circ} \mathrm{C}$. The buffer blank was performed under the same conditions and showed negligible heats of binding. The dissociation constant $\left(K_{\mathrm{d}}\right)$, stoichiometry of binding $(n)$, binding enthalpy $(\Delta H)$, and entropy $(\Delta S)$ were obtained by fitting the data to a single-site binding model with the data analysis software (Origin 7) provided by the manufacturer. Baselines were subtracted from final data using the Origin software. For the full-length $\mathrm{Neh} 2$ experiments, the data were fitted either to a two-site binding model (wild-type Kelch, G350S, and A427V) or a one-site-binding model (G364C and R415G). Protein concentrations were confirmed by amino acid analysis (Advanced Protein Technology Centre, The Hospital for Sick Children, Toronto, ON). 


\section{RESULTS}

\section{Mutations exert differential effects on the solubility of the Kelch domain of Keap1}

The wild-type Kelch domain of human Keapl was over-expressed in E. coli and purified by the procedure described previously [31]. Four of the Kelch mutants, G350S, G364C, R415G and A427V, were found to have similar solubility as the wild-type and were purified with comparable yields. The R413L, G333C, G379D, G430C and G476R mutants, on the other hand, were found almost exclusively in the insoluble fractions under various over-expression conditions tested. We selected to purify the G333C, G379D, and the R413L proteins from inclusion bodies using $8 \mathrm{M}$ Urea and subjected to refolding. Severe protein aggregation occurred upon refolding and only a small amount of soluble protein was obtained for the three mutants. The signals observed in the ${ }^{1} \mathrm{H}^{15} \mathrm{~N}$ HSQC spectra (Figures S1A \& B) of G333C and G379D proteins were broad and poorly dispersed in the proton dimension, suggesting that both mutants were aggregated. While the ${ }^{1} \mathrm{H}-{ }^{15} \mathrm{~N}$ HSQC spectrum of R413L (Figure S1C) resembles that of a folded protein; however, we were unable to obtain a stable (even at $4{ }^{\circ} \mathrm{C}$ ) high purity protein sample.

\section{Probing structural changes of the Kelch domain upon mutations by CD and NMR}

CD and NMR were used to identify the effects of missense mutations on the overall folding and local conformation of the Kelch domain. The CD results revealed that the $\beta$-sheet secondary structure content, observed in the wild-type Kelch domain, is preserved in G364C, G350S, R415G and A427V (Figure S2). The percentages of $\beta$-sheet and disordered regions estimated from the deconvolution of CD spectra are almost identical to the wild-type protein (Table S2), strongly suggesting that these four mutations do not have a significant effect on the overall fold of the Kelch domain.

We then used NMR spectroscopy to investigate the effects of somatic mutations on the local structural changes and ligand-binding of the Kelch domain. To be able to monitor the site-specific conformational changes upon mutation and target binding, backbone chemical shift assignment of the 34-kDa human Kelch domain was performed. ${ }^{1} \mathrm{HN},{ }^{15} \mathrm{~N},{ }^{13} \mathrm{C} \alpha,{ }^{13} \mathrm{C} \beta$ resonance signals were assigned using the HNCACB, $\mathrm{HN}(\mathrm{CO}) \mathrm{CACB}$, and ${ }^{15} \mathrm{~N}$ NOESY-HSQC experiments. The assigned ${ }^{1} \mathrm{H}-{ }^{15} \mathrm{~N}$ TROSYHSQC spectrum is showed in Figure 2A. Although the amino acid sequence of Kelch contains regions of degeneracy (Figure 2B), we were able to assign $92 \%$ of the ${ }^{1} \mathrm{HN}$ and ${ }^{15} \mathrm{~N}$ resonances of the non-proline residues, $91.6 \%$ of ${ }^{13} \mathrm{C} \alpha$ and $90.7 \%$ of ${ }^{13} \mathrm{C} \beta$ of all residues. The chemical shift assignments of the human Kelch domain are comparable to the assignments of the Kelch domain of mouse Keap1 that we have completed previously [43].

To probe the conformational changes of Kelch upon mutations, ${ }^{1} \mathrm{H}-{ }^{15} \mathrm{~N}$ TROSYHSQCs of G364C, R415G, G350S, and A427V were collected and compared to the spectrum of wild-type Kelch (Figures S3A-D). The dispersion of the amide peaks for all four mutants is similar to that of the wild-type Kelch, indicating that the mutants remain well-folded. Further analysis of the chemical shift perturbations (Figure 3) revealed that A427V displayed the largest amplitude of peak shifts followed by G364C, R415G and G350S. It is noteworthy that large chemical shift changes are observed for some residues that are located far from the mutation sites in the protein sequence for A427V, G364C 
and R415G mutants. For instance, A427 is located in the middle of the second $\beta$-strand of Blade III. Mutating of this residue to a valine caused peak shifts of a significant number of residues clustered in Blade II (residues V360-M409) and Blade III (S410L457), in particular, S363, G379, E446 and A466 residues (Figure 3C). The S363 and G379 residues also undergo a substantial change in the G364C spectrum (Figure 3B).

\section{Effects of mutations on the target recognition of the Kelch domain}

We used NMR and ITC to assess the effects of mutations (G350S, G364C, $\mathrm{R} 415 \mathrm{G}$ and $\mathrm{A} 427 \mathrm{~V}$ ) on the target recognition of the Kelch domain of Keap1. First, peptides encoding the previously reported Neh2-ETGE (residues 69-84) [38], Neh2-DLG (residues 20-39) [41], and ProT $\alpha$-ENGE (residues 33-52) [31] Kelch-binding motifs were used in the studies. NMR experiments allowed to elucidate the relative contributions from Kelch residues (wild type and mutants) involved in the interactions with Neh2-ETGE, Neh2-DLG, and ProT $\alpha$-ENGE. Figures $4 \mathrm{~A} \& \mathrm{C}$ show the ${ }^{1} \mathrm{H}-{ }^{15} \mathrm{~N}$ HSQC spectra of the wild-type Kelch domain in the presence of 5 molar equivalents of unlabeled Neh2-ETGE and ProT $\alpha$-ENGE peptides, respectively. Quantitative analysis of the Kelch chemical shift perturbations revealed large changes in S363, G417, G462, S508, G527, and T576 in the Neh2-ETGE and ProT $\alpha$-ENGE bound state (Figures 5A \& C). Some of these residues are directly involved in complex formation with targets, whereas the peak shift of others may be due to the changes in their local chemical environment [36, 40, 41]. For example, S363 was found to form a hydrogen bond with the E46 of ProT $\alpha$ in the complex [40]. Meanwhile, the G462 residue does not interact with ProT $\alpha$ directly, rather it forms a intramolecular hydrogen bond with R415 which is involved in a salt bridge with E43 of ProT $\alpha$ [40].

The amplitudes of chemical shift changes of G350S and A427V upon binding to the Neh2-ETGE and ProT $\alpha$-ENGE peptides were comparable to those of the wild-type (Figures 5A \& C, Figure S4C \& D, Figure S5C \& D). G364C and R415G displayed smaller amplitudes of chemical shift perturbations upon binding to the Neh2-ETGE and ProT $\alpha$-ENGE peptides (Figures 5B \& D, Figures S4A \& B, Figures S5A \& B). Despite the difference in the amplitudes of changes, the overall chemical shift perturbation pattern is similar to the wild-type, suggesting that in the presence of either one of these two mutations, Kelch can still bind to the Neh2-ETGE and ProT $\alpha$-ENGE peptides in similar modes, but likely with lower affinities.

Meanwhile, titration of the Neh2-DLG peptide to the wild-type Kelch domain (Figures 4B \& 5E) results in overall smaller chemical shift changes of the protein, suggesting that the Neh2-DLG peptide only forms weak interactions with Kelch. Noticeable resonance shifts were observed for R354, D385, H436 and A607. The H436 residue was found to interact with the conserved G430 residue. The chemical environment of the G430 is influenced by the R415 residue, which takes part in hydrogen bonding with the targets [41]. Similarly, the chemical environment of other Kelch residues undergoing large peak shifts are affected by amino acids involved in hydrogen bonding with targets. 
Figures S6A-D show the ${ }^{1} \mathrm{H}_{-}{ }^{15} \mathrm{~N}$ HSQC spectra of G364C, R415G, G350S, and A427V in the absence and presence of five molar equivalents of the Neh2-DLG peptides. For the G350S and A427V mutants, while many resonance signals broaden out in the ligand-bound state, both mutants display similar chemical shift attenuation patterns as the wild-type Kelch. Meanwhile, the relatively minor chemical shift changes observed for the G364C and R415G upon addition of DLG peptides indicate that the complex formation is severely disrupted by the mutations (Figure 5F and Figures S6A \& B). In fact, for the $\mathrm{G} 364 \mathrm{C}$, we observe no significant peak broadening and only minor resonance perturbations across the whole spectrum. The minor shifts observed in the bound state NMR spectra of G364C-DLG complex and R415G-DLG complex suggest that the presence of either one of these two somatic mutations in the Kelch domain can depress the ability of Keap1 to interact with the DLG motif of Neh2.

We then used ITC to further quantify the effects of mutations on the target binding of the Kelch domain. Table 1 lists the binding parameters of the wild-type and mutants of the Kelch domain to the ProT $\alpha$-ENGE and Neh2-ETGE peptides (ITC profiles are shown in Figure S7). The dissociation constant $\left(K_{d}\right)$ of the wild-type Kelch/ProT $\alpha$-ENGE and Kelch/Neh2-ETGE complexes were determined to be $2.7 \pm 0.1$ $\mu \mathrm{M}$ and $31 \pm 2 \mathrm{nM}$, respectively. These values are on par with the values of $\sim 2.6 \mu \mathrm{M}$ and $\sim 20 \mathrm{nM}$ for Kelch complex formation with full-length ProT $\alpha$ and with a 16-mer Neh2ETGE peptide as reported in the literature $[31,36]$. The binding affinities of the mutants to ProT $\alpha$ and Neh2-ETGE peptides varied. While the G350S mutation has only minor effects on the target binding $(3.0 \pm 0.1 \mu \mathrm{M}$ for the ProT $\alpha$-ENGE and to $38 \pm 4 \mathrm{nM}$ for the Neh2-ETGE peptides), the G364C mutation lowered the binding affinity for both Neh2ETGE and ProT $\alpha$-ENGE peptides by $>10$ fold compared to the wild-type complex $(31 \pm$ $2 \mu \mathrm{M}$ for the ProT $\alpha$-ENGE and to $345 \pm 12 \mathrm{nM}$ for the Neh2-ETGE peptides). Our ITC data show that the interactions of G364C with targets are enthalpically less favorable compared to the wild-type interactions. Replacement of the Gly residue with a Cys may restrict the conformational freedom of the neighboring region and/or possibly disrupt the wild-type hydrogen bond network.

The binding affinities of Kelch to both the ProT $\alpha$-ENGE and Neh2-ETGE peptides were severely impaired by the R415G mutation to an extent that the affinities could not be accurately determined by ITC (Figures S7D \& I). Intriguingly, in contrast to G350S, G364C and R415G, the interactions with ProT $\alpha$-ENGE and Neh2-ETGE peptides were modestly strengthened with the A427V mutation. The $K_{\mathrm{d}}$ values are $1.90 \pm$ $0.04 \mu \mathrm{M}$ and $16 \pm 2 \mathrm{nM}$, respectively (Table 1). Interestingly, A427V/ProT $\alpha$-ENGE complex formation was enthalpically more favorable, while the entropy change is more negative in comparison with the wild-type.

We also attempted to measure the binding affinities of a 20-residue Neh2-DLG peptide (encoding the sequence of I20-F39 of Nrf2) [41] to the wild-type Kelch domain (Figure S7K) and the four mutants; however, no binding was detected by ITC at $25^{\circ} \mathrm{C}$ in any case. NMR titration experiments, which were performed with higher concentrations of protein and peptide, however showed that Neh2-DLG is capable of forming complex with the Kelch domain (Figure 4B), likely with low binding affinity. Our results 
suggested that either the interaction between the Kelch domain and the Neh2-DLG peptide is very weak for ITC measurement or the $\Delta H$ of the binding is close to zero. No detectable binding was observed at 5 and $35^{\circ} \mathrm{C}$ either (data not shown). This observation is in good agreement with the results obtained very recently by Fukutomi et al. [47] showing that the binding of the Kelch domain with a 16-mer peptide containing the previously defined DLG motif of Nrf2 (residues 22-37) was too weak to be detected by differential scanning fluorimetry. Intriguingly, in the same study and experiment, binding was detected using an extended DLG peptide comprising residues 17-51 (DLGex) of Nrf2, which suggests that residues flanking the DLG motif are critical for binding to Kelch.

We investigated the binding of the Kelch domain to the DLG site in the context of full-length Neh2 using a Neh2 $\triangle$ ETGE construct (deletion of ${ }^{79} \mathrm{ETGE}^{82}$ from full-length Neh2). Notably, NMR binding experiments of the Neh2 $\triangle$ ETGE with the Kelch domain reveal significant chemical perturbations in the N-terminal residues 6 to 40 of Neh2 $\triangle$ ETGE (Figure S8). Resonance broadening occurring in a large number of flanking residues supports the findings by Fukutomi et al. [47] that the DLG motif is indeed longer than previously defined [41]. Figures S9A-E show the isotherms for the binding of the Neh2 $\Delta$ ETGE protein to the Kelch domain at $25{ }^{\circ} \mathrm{C}, 20{ }^{\circ} \mathrm{C}, 15{ }^{\circ} \mathrm{C}, 10{ }^{\circ} \mathrm{C}$ and $5{ }^{\circ} \mathrm{C}$, respectively. It is apparent that the complex formation is endothermic at low temperatures and as the temperature increases, the enthalpy change becomes smaller. Table S4 lists the thermodynamic parameters for the Neh2 $\Delta$ ETGE-Kelch complex at 5 ${ }^{\circ} \mathrm{C}$ and $10{ }^{\circ} \mathrm{C}$. With the buffer conditions used in this study, the $K_{d}$ of the human Neh2 $\triangle$ ETGE/Kelch binding was determined to be $\sim 351 \pm 16 \mathrm{nM}$ at $10{ }^{\circ} \mathrm{C}$. The value is similar to what was determined for the mouse Kelch binding to the Neh2 $\triangle \mathrm{ETGE}\left(K_{d}\right.$ $\sim 128 \mathrm{nM}$ ) [47], as well as to DLGex peptide (residues $17-51, K_{d} \sim 526 \mathrm{nM}$ ) at $25^{\circ} \mathrm{C}$ [47]. We then estimated the $\Delta C_{p}$ value based on the $\Delta H$ values at the $5{ }^{\circ} \mathrm{C}$ and $10{ }^{\circ} \mathrm{C}$. Based on the calculated $\Delta C_{p}$ value of $-0.47 \mathrm{kcal} \mathrm{mol}^{-1} \mathrm{~K}^{-1}$, the $\Delta H$ at $25^{\circ} \mathrm{C}$ was estimated to be $\sim-1.2 \mathrm{kcal} / \mathrm{mol}$. This small value of enthalpy change explains why binding between the Neh2 $\triangle$ ETGE and the Kelch domain could not be observed at $25^{\circ} \mathrm{C}$ by ITC.

\section{Somatic mutations in the Kelch domain affect its interaction with the full-length Neh2 domain}

To examine how the somatic mutations in the Kelch domain affect its interactions with the DLG and ETGE sites in the context of full-length Neh2, ITC experiments were carried out at $5{ }^{\circ} \mathrm{C}$ to determine the binding parameters of the wild-type Kelch and four mutational variants with the Neh2 domain (residues 1-98) of Nrf2. Kelch proteins were titrated to the full-length Neh2 and the ITC isotherms were fit to either a two-site or onesite binding model. The results are listed in Table 2 (Figures S9F-J shows the ITC isotherms). Similar to what was observed for the binding of the Kelch domain to the Neh2-ETGE peptide, both R415G and G364C mutations dramatically weakened Kelch's interaction with the ETGE site of the full-length Neh2. Compared to the wild-type Kelch, which binds the ETGE site with a $K_{d}$ of $1.26 \pm 0.03 \mathrm{nM}, \mathrm{G} 364 \mathrm{C}$ and R415G have binding affinities that are 37 and 870 fold lower, respectively. On the other hand, the G350S and A427V mutations show no significant impact on the ETGE binding. It is noteworthy that the binding affinities of the Neh2-ETGE motif to the wild-type and 
mutational variants of Kelch are tighter when present in the full-length Neh2 protein as opposed to a peptide. For instance, the Kelch R $415 \mathrm{G}$ provided a measurable affinity ( $K_{d}$ of $1.1 \mu \mathrm{M})$ when titrated into full-length Neh2 but could not be accurately determined for the ETGE peptide (Figures S7I \& S9I).

As also observed for the mouse Kelch-DLG complexes [30,47], the DLG site in the context of full-length human Neh2 binds significantly weaker to the Kelch domain compared to the ETGE site $\left(\mathrm{K}_{\mathrm{d}}\right.$ of $179 \mathrm{nM}$ vs $\left.1.26 \mathrm{nM}\right)$. For the mutants, the binding affinity of the G350S to the DLG site decreased by less than two-fold while the A427V mutant has the same affinity ( $K_{d}$ of $179 \pm 4 \mathrm{nM}$ ) as compared to the wild-type. Our ITC data is in accordance with the NMR results, indicating that in the presence of either A427V or G350S mutations, the Kelch domain should still be able to interact effectively with both the ETGE and DLG sites of Nrf2. On the other hand, the interactions of the DLG site with G364C and R415G could not be detected by ITC (Figures S9H \& I) even in the context of full length Neh2, while the ETGE site interacts with R415G and G364C but with lower affinities. 


\section{DISCUSSION}

In this work, we have characterized the effects of cancer-associated somatic mutations on structure and target recognition of Keap1. Nine mutations identified in lung cancer patients or cancer cell lines, were studied based on their location in the Kelch domain (Table S1). ITC, CD and NMR techniques were used to quantify the binding parameters of these mutational variants with two of the binding targets of Keap1 and to assess the conformational changes these mutations induce on the Kelch structure. Our results indicated that the disease-linked somatic mutations have differential effects on the structural integrity and the target recognition of Keap1.

The G333, G379, G430, G476 and R413 residues are conserved in the Kelch domain. Our protein expression, purification and NMR data (Figures S1) suggest that mutating these residues can result in the unfolding/misfolding of the Kelch domain. Further in vivo experiments are required to verify these findings. As mentioned earlier, the G333, G379, G430 and G476 all play key roles in the folding of the Kelch domain [39]. The crystal structure of the human Kelch domain (PDB: 1U6D) shows that the backbone torsion angles of these four glycines are generally located in disallowed regions. Therefore, mutating these residues to any other amino acid with a $\beta$-carbon side chain may induce severe conformational strain in the structure, causing the protein to unfold/misfold. Previous studies using immunoprecipitation and luciferase assays have demonstrated that the G333C, G379D, and G430C mutants are defective in repressing Nrf2-mediated transcription of cytoprotective genes $[12,15,16]$. In agreement with these findings, our results suggest that reduction in protein stability of Keap1 is the cause of the loss-of-function of these mutants. We speculate that any other mutations occurring in the double glycine repeats (e.g. G333S and G524C listed in the COSMIC database) will be detrimental to Kelch's structural integrity $[12,15,16]$.

Although the ${ }^{1} \mathrm{H}-{ }^{15} \mathrm{~N}$ HSQC spectrum of Kelch R413L resembled that of a folded protein (Figure S1C), the mutant is not stable compared to the wild-type Kelch. R413, located in Blade III, is one of the conserved Arg residues found in all six blades of the $\beta$ propeller structure [39]. The side-chain of this residue is involved in inter-blade hydrogen bonding with G379, the first residue of the glycine doublet in Blade II [39]. It has been suggested that the inter-blade hydrogen bonding between these conserved Arg and Gly residues are important for maintaining the Kelch structure [39]. Our results demonstrated that a single point mutation in the R413 position is sufficient to destabilize the protein, and probably leads to the loss of function. The impacts of mutations of other conserved Arg residues (e.g. R362Q, R460G, R460S, R554Q [32], and R601W listed in COSMIC) on the protein stability remain to be elucidated.

Unlike the G333C, G379D, R413L, G430C, and G476R mutants, G350S, G364C, $\mathrm{R} 415 \mathrm{G}$, and $\mathrm{A} 427 \mathrm{~V}$ have comparable solubility to the wild-type protein. CD analysis of these four mutational variants illustrated that they all possess similar secondary structure contents as the wild-type (Figure S2). On the other hand, our ITC results clearly demonstrated that, depending on their locations in the protein, these four mutations exert differential impacts on the target binding of the Kelch domain (Tables 1 \& 2). The G350 residue is located in a loop region at the opposite face of the ligand-binding pocket of the 
$\beta$-propeller structure, forming a hydrogen bond with the neighboring N346 residue [39]. Crystal structures of the Kelch domain in complex with the Neh2-ETGE, Neh2-DLG, and ProT $\alpha$-ENGE peptides revealed that this residue was not directly involved in the interactions with targets $[36,40,41]$. Even though the G350S mutation has been identified in non-small-cell lung cancer cell lines [12] and gastric cancer tissue [33], the mutation appears to cause no substantial changes to the binding of ProT $\alpha$ or the Neh2 domain of Nrf2: only a 1-2 fold decrease in the binding affinity to ProT $\alpha$-ENGE, Neh2ETGE, and Neh2-DLG motifs compared to the wild-type Kelch were observed. NMR data further demonstrate that the G350S mutation does not lead to any significant change in the protein structure. Together, our results suggest that this particular mutation does not hamper the complex formation of Keap1 with Nrf2 and ProT $\alpha$. Effects of this mutation on the interactions of Keap1 with other targets, however, remain to be investigated $[32,42]$.

The Kelch A427V is another cancer-linked mutation (identified in cancer tissue of a patient with small cell carcinoma [16]) that was observed to interact with Nrf2 and ProT $\alpha$ with similar affinities to wild-type Kelch. In fact, this particular Ala to Val substitution resulted in modest increases $(\sim 2$ fold) in the binding affinity of Kelch to ProT $\alpha$-ENGE and Neh2-ETGE peptides (Table 1). Our NMR results further confirm that the binding of this mutant to ProT $\alpha$-ENGE, Neh2-ETGE and Neh2-DLG motifs are similar to the wild-type interactions with targets. Intriguingly, while the G350S mutation resulted in subtle chemical shift changes to residues around the mutation site (Figure $3 \mathrm{~A}$ ), the A427V mutation, found in the $\beta 2$ strand of blade III, leads to local structural changes in blades II and III, which are reflected by the substantial chemical shift changes observed (Figure 3C). However, these local structural changes do not impair the binding of Kelch to either Neh2 or ProT $\alpha$. Since the Kelch domain of Keap1 also mediates the interaction with WTX [22], p62 [23], PGAM5 [24], PALB2 [25], FAC1 [26], IKK $\beta$ [27], and many other proteins [28], characterizing the effect of the A427V mutation on the binding of these targets will provide further insight into the links of this particular somatic mutation to the associated disease. It is noteworthy that Hast et al. [32] recently reported that the R470C, D422N, and G423V mutations also increase the binding to Nrf2 compared to the wild-type Keap1 based on their immunoprecipitation and Western blot analysis. However, the molecular effects of these cancer-associated mutations on the structure of Kelch remain unknown. Interestingly, Baird et al. have demonstrated that sulforaphane, a well-known Nrf2-inducer, can enhance the interaction between Keap1 and Nrf2 by provoking conformational changes to the complex [48]. Despite the tighter interaction between these two proteins, it has been suggested that Nrf2 is not properly oriented in the induced state for ubiquitination by the E2 ubiquitin-conjugating enzyme [48]. In light of this finding, it is possible that some of these tighter binding mutants may induce subtle changes to the mode of Keap1-Nrf2 interaction, rendering the ubiquitination/degradation of Nrf2 ineffective [32, 48].

Meanwhile, our ITC and NMR results show that both the G364C and R415G mutations impair Kelch's ability to interact with Nrf2 and ProT $\alpha$, albeit to different extents. The G364C substitution resulted in significantly lower affinity for both Neh2ETGE and ProT $\alpha$-ENGE peptides $\left(K_{d}(\mathrm{Neh} 2-\mathrm{ETGE})=345 \mathrm{nM}\right.$ and $K_{d}(\operatorname{ProT} \alpha-\mathrm{ENGE})=$ 
$31 \mu \mathrm{M}$ vs $K_{d}(\mathrm{Neh} 2-\mathrm{ETGE})=31 \mathrm{nM}$ and $K_{d}(\operatorname{ProT\alpha }-\mathrm{ENGE})=2.7 \mu \mathrm{M}$ for the wild-type Kelch). The substantial decrease in affinity for the ETGE motif was also observed in the context of full-length Neh2 domain ( $K_{d}=47 \mathrm{nM}$ vs $K_{d}=1.26 \mathrm{nM}$ for wild-type Kelch). The crystal structure of the human Kelch domain in complex with the ETGE peptide illustrates that G364 has $\phi / \psi$ torsion angles of left-handed helix and is not involved in hydrogen bonding with the target. However, this Gly residue is located next to S363, which forms hydrogen bonds with E82 of the ETGE peptides [36, 40]. Replacing the Gly with a Cys residue may restrict the orientation of the S363 side chain from forming hydrogen bonds with E82. Additionally, the G364C mutation may also affect the intramolecular hydrogen bonding with the G603 residue [39]. It is noteworthy that even though the G364C and R415G mutations significantly weaken the interactions of Kelch with ProT $\alpha$-ENGE and the Neh2-ETGE motifs, low binding affinities with these two targets could still be measured. On the other hand, no interaction between these two mutants and the DLG motif (even in the context of full-length Neh2) could be detected by ITC.

The R415 residue of Kelch is directly involved in the binding with targets [23, 36, 40]. In the mouse Keap1-ProT $\alpha$ peptide complex, R415 is involved in electrostatic interactions with the E43 residue of ProT $\alpha$ [40]. This Arg residue also forms salt bridges with the E79 residue in the binding interface with the Neh2-ETGE peptide [36]. As such, it is not a surprise that replacing this critical Arg residue in the positively charged pocket with a non-polar Gly can significantly weaken the interactions of the Kelch domain with its targets, and this was observed in our ITC and NMR binding studies with ProT $\alpha$ and Neh2. In agreement with this, a study by Tong et al. [30], which replaced R415 with a Lys also attenuated the binding of Kelch to the ETGE motif of Neh2 domain. Similarly, Lo et al. [36] detected no Nrf2 repressive activity for the R415A mutant. We speculate that mutations of other residues that are in direct contact with the ETGE and DLG motifs of Neh2 (e.g. Y334H, R483C, S555C, and Y572C) will ultimately impair the interaction of Keap1 with Nrf2. Further in vivo and in vitro experiments will allow correlating the molecular effects of different categories of mutations with their impacts on the Nrf2repression function systematically. 


\begin{abstract}
AUTHOR CONTRIBUTIONS
Halema Khan and Wing-Yiu Choy conceived and designed the experiments. Halema Khan, Ryan Killoran and Anne Brickenden performed the experiments. Jingsong Fan and Daiwen Yang performed the 3D backbone resonance assignment NMR experiments on the human Kelch domain. Halema Khan analyzed the data. Halema Khan and WingYiu Choy wrote the paper.
\end{abstract}

\title{
ACKNOWLEDGEMENTS
}

We thank the Biomolecular NMR Facility and the Bimolecular Interaction and Conformation Facility for their assistance and use of the facilities. We would also like to acknowledge the support of the NMR facility at the National University of Singapore.

\section{FUNDING}

This work was supported by an Operating Grant (MOP 74679) from the Canadian Institutes of Health Research (CIHR). 


\section{REFERENCES}

1 Jaramillo, M. C. and Zhang, D. D. (2013) The emerging role of the Nrf2-Keap1 signaling pathway in cancer. Genes Dev. 27, 2179-2191

2 Müller, T. and Hengstermann, A. (2012) Nrf2: Friend and Foe in Preventing Cigarette Smoking-Dependent Lung Disease. Chem. Res. Toxicol. 25, 1805-1824

3 Kwak, M.-K. and Kensler, T. W. (2010) Targeting NRF2 signaling for cancer chemoprevention. Toxicol. Appl. Pharmacol. 244, 66-76

4 Suzuki, T., Motohashi, H. and Yamamoto, M. (2013) Toward clinical application of the Keap1-Nrf2 pathway. Trends Pharmacol. Sci. 34, 340-346

5 Itoh, K., Wakabayashi, N., Katoh, Y., Ishii, T., Igarashi, K., Engel, J. D. and Yamamoto, M. (1999) Keap1 represses nuclear activation of antioxidant responsive elements by Nrf2 through binding to the amino-terminal Neh2 domain. Genes Dev. 13, 76-86

6 Itoh, K., Chiba, T., Takahashi, S., Ishii, T., Igarashi, K., Katoh, Y., Oyake, T., Hayashi, N., Satoh, K., Hatakeyama, I., Yamamoto, M. and Nabeshima, Y.-I. (1997) An Nrf2/Small Maf Heterodimer Mediates the Induction of Phase II Detoxifying Enzyme Genes through Antioxidant Response Elements. Biochem. Biophys. Res. Commun. 236, 313-322

7 Zhang, D. D., Lo, S. C., Cross, J. V., Templeton, D. J. and Hannink, M. (2004) Keap1 is a redox-regulated substrate adaptor protein for a Cul3-dependent ubiquitin ligase complex. Mol. Cell. Biol. 24, 10941-10953

8 Kobayashi, A., Kang, M. I., Okawa, H., Ohtsuji, M., Zenke, Y., Chiba, T., Igarashi, K. and Yamamoto, M. (2004) Oxidative stress sensor Keap1 functions as an adaptor for Cul3-based E3 ligase to regulate proteasomal degradation of Nrf2. Mol. Cell. Biol. 24, 7130-7139

9 McMahon, M., Thomas, N., Itoh, K., Yamamoto, M. and Hayes, J. D. (2006) Dimerization of substrate adaptors can facilitate cullin-mediated ubiquitylation of proteins by a "tethering" mechanism: a two-site interaction model for the Nrf2-Keap1 complex. J. Biol. Chem. 281, 24756-24768

10 Zhang, D. D. and Hannink, M. (2003) Distinct cysteine residues in Keap1 are required for Keap1-dependent ubiquitination of $\mathrm{Nrf2}$ and for stabilization of $\mathrm{Nrf2}$ by chemopreventive agents and oxidative stress. Mol. Cell. Biol. 23, 8137-8151

11 Hayes, J. D. and McMahon, M. (2009) NRF2 and KEAP1 mutations: permanent activation of an adaptive response in cancer. Trends. Biochem. Sci. 34, 176-188

12 Singh, A., Misra, V., Thimmulappa, R. K., Lee, H., Ames, S., Hoque, M. O., Herman, J. G., Baylin, S. B., Sidransky, D., Gabrielson, E., Brock, M. V. and Biswal, S. 
(2006) Dysfunctional KEAP1-NRF2 interaction in non-small-cell lung cancer. PLoS Med. 3, e420

13 McMahon, M., Itoh, K., Yamamoto, M. and Hayes, J. D. (2003) Keap1-dependent proteasomal degradation of transcription factor Nrf2 contributes to the negative regulation of antioxidant response element-driven gene expression. J. Biol. Chem. 278, $21592-21600$

14 Niture, S. K. and Jaiswal, A. K. (2009) Prothymosin-\{alpha $\}$ Mediates Nuclear Import of the INrf2/Cul3-Rbx1 Complex to Degrade Nuclear Nrf2. J. Biol. Chem. 284, $13856-13868$

15 Shibata, T., Kokubu, A., Gotoh, M., Ojima, H., Ohta, T., Yamamoto, M. and Hirohashi, S. (2008) Genetic alteration of Keap1 confers constitutive Nrf2 activation and resistance to chemotherapy in gallbladder cancer. Gastroenterology. 135, 1358-1368

16 Ohta, T., Iijima, K., Miyamoto, M., Nakahara, I., Tanaka, H., Ohtsuji, M., Suzuki, T., Kobayashi, A., Yokota, J., Sakiyama, T., Shibata, T., Yamamoto, M. and Hirohashi, S. (2008) Loss of Keap1 function activates Nrf2 and provides advantages for lung cancer cell growth. Cancer Res. 68, 1303-1309

17 Nioi, P. and Nguyen, T. (2007) A mutation of Keap1 found in breast cancer impairs its ability to repress Nrf2 activity. Biochem. Biophys. Res. Commun. 362, 816821

18 Fourquet, S., Guerois, R., Biard, D. and Toledano, M. B. (2010) Activation of NRF2 by nitrosative agents and $\mathrm{H} 2 \mathrm{O} 2$ involves KEAP1 disulfide formation. J. Biol. Chem. 285, 8463-8471

19 McMahon, M., Lamont, D. J., Beattie, K. A. and Hayes, J. D. (2010) Keap1 perceives stress via three sensors for the endogenous signaling molecules nitric oxide, zinc, and alkenals. Proc. Natl. Acad. Sci. U. S. A. 107, 18838-18843

20 Dinkova-Kostova, A. T., Holtzclaw, W. D., Cole, R. N., Itoh, K., Wakabayashi, N., Katoh, Y., Yamamoto, M. and Talalay, P. (2002) Direct evidence that sulfhydryl groups of Keap1 are the sensors regulating induction of phase 2 enzymes that protect against carcinogens and oxidants. Proc. Natl. Acad. Sci. U. S. A. 99, 11908-11913

21 Cullinan, S. B., Gordan, J. D., Jin, J., Harper, J. W. and Diehl, J. A. (2004) The Keap1-BTB protein is an adaptor that bridges Nrf2 to a Cul3-based E3 ligase: oxidative stress sensing by a Cul3-Keap1 ligase. Mol. Cell. Biol. 24, 8477-8486

22 Camp, N. D., James, R. G., Dawson, D. W., Yan, F., Davison, J. M., Houck, S. A., Tang, X., Zheng, N., Major, M. B. and Moon, R. T. (2012) Wilms tumor gene on X chromosome (WTX) inhibits degradation of NRF2 protein through competitive binding to KEAP1 protein. J. Biol. Chem. 287, 6539-6550 
23 Komatsu, M., Kurokawa, H., Waguri, S., Taguchi, K., Kobayashi, A., Ichimura, Y., Sou, Y.-S., Ueno, I., Sakamoto, A., Tong, K. I., Kim, M., Nishito, Y., Iemura, S.-i., Natsume, T., Ueno, T., Kominami, E., Motohashi, H., Tanaka, K. and Yamamoto, M. (2010) The selective autophagy substrate $\mathrm{p} 62$ activates the stress responsive transcription factor Nrf2 through inactivation of Keap1. Nat. Cell. Biol. 12, 213-223

24 Lo, S.-C. and Hannink, M. (2006) PGAM5, a Bcl-XL-interacting protein, is a novel substrate for the redox-regulated Keap1-dependent ubiquitin ligase complex. J. Biol. Chem. 281, 37893-37903

25 Ma, J., Cai, H., Wu, T., Sobhian, B., Huo, Y., Alcivar, A., Mehta, M., Cheung, K. L., Ganesan, S., Kong, A. N. T., Zhang, D. D. and Xia, B. (2012) PALB2 Interacts with KEAP1 To Promote NRF2 Nuclear Accumulation and Function. Mol. Cell. Biol. 32, 1506-1517

26 Strachan, G. D., Morgan, K. L., Otis, L. L., Caltagarone, J., Gittis, A., Bowser, R. and Jordan-Sciutto, K. L. (2004) Fetal Alz-50 clone 1 interacts with the human orthologue of the Kelch-like Ech-associated protein. Biochemistry. 43, 12113-12122

27 Kim, J.-E., You, D.-J., Lee, C., Ahn, C., Seong, J. Y. and Hwang, J.-I. (2010) Suppression of NF-kappaB signaling by KEAP1 regulation of IKKbeta activity through autophagic degradation and inhibition of phosphorylation. Cell Signal. 22, 1645-1654

28 Hast, B. E., Goldfarb, D., Mulvaney, K. M., Hast, M. A., Siesser, P. F., Yan, F., Hayes, D. N. and Major, M. B. (2013) Proteomic analysis of ubiquitin ligase KEAP1 reveals associated proteins that inhibit NRF2 ubiquitination. Cancer Res. 73, 2199-2210

29 Tong, K. I., Kobayashi, A., Katsuoka, F. and Yamamoto, M. (2006) Two-site substrate recognition model for the Keap1-Nrf2 system: a hinge and latch mechanism. Biol. Chem. 387, 1311-1320

30 Tong, K. I., Katoh, Y., Kusunoki, H., Itoh, K., Tanaka, T. and Yamamoto, M. (2006) Keap1 recruits Neh2 through binding to ETGE and DLG motifs: characterization of the two-site molecular recognition model. Mol. Cell. Biol. 26, 2887-2900

31 Khan, H., Cino, E. A., Brickenden, A., Fan, J., Yang, D. and Choy, W. Y. (2013) Fuzzy Complex Formation between the Intrinsically Disordered Prothymosin alpha and the Kelch Domain of Keap1 Involved in the Oxidative Stress Response. J. Mol. Biol. 425, 1011-1027

32 Hast, B. E., Cloer, E. W., Goldfarb, D., Li, H., Siesser, P. F., Yan, F., Walter, V., Zheng, N., Hayes, D. N. and Major, M. B. (2014) Cancer-derived mutations in KEAP1 impair NRF2 degradation but not ubiquitination. Cancer Res. 74, 808-817

33 Yoo, N. J., Kim, H. R., Kim, Y. R., An, C. H. and Lee, S. H. (2012) Somatic mutations of the KEAP1 gene in common solid cancers. Histopathology. 60, 943-952 
34 The Cancer Genome Atlas Research Network (2012) Comprehensive genomic characterization of squamous cell lung cancers. Nature. 489, 519-525

35 Suzuki, T., Maher, J. and Yamamoto, M. (2011) Select heterozygous Keap1 mutations have a dominant-negative effect on wild-type Keap1 in vivo. Cancer Res. 71, 1700-1709

36 Lo, S.-C., Li, X., Henzl, M. T., Beamer, L. J. and Hannink, M. (2006) Structure of the Keap1:Nrf2 interface provides mechanistic insight into Nrf2 signaling. EMBO J. 25, 3605-3617

37 Forbes, S. A., Bindal, N., Bamford, S., Cole, C., Kok, C. Y., Beare, D., Jia, M., Shepherd, R., Leung, K., Menzies, A., Teague, J. W., Campbell, P. J., Stratton, M. R. and Futreal, P. A. (2011) COSMIC: mining complete cancer genomes in the Catalogue of Somatic Mutations in Cancer. Nucleic Acids Res. 39, D945-950

38 Padmanabhan, B., Tong, K. I., Ohta, T., Nakamura, Y., Scharlock, M., Ohtsuji, M., Kang, M. I., Kobayashi, A., Yokoyama, S. and Yamamoto, M. (2006) Structural basis for defects of Keap1 activity provoked by its point mutations in lung cancer. Mol. Cell. 21, 689-700

39 Li, X., Zhang, D., Hannink, M. and Beamer, L. J. (2004) Crystal structure of the Kelch domain of human Keap1. J. Biol. Chem. 279, 54750-54758

40 Padmanabhan, B., Nakamura, Y. and Yokoyama, S. (2008) Structural analysis of the complex of Keap1 with a prothymosin alpha peptide. Acta Cryst. F. 64, 233-238

41 Tong, K. I., Padmanabhan, B., Kobayashi, A., Shang, C., Hirotsu, Y., Yokoyama, S. and Yamamoto, M. (2007) Different electrostatic potentials define ETGE and DLG motifs as hinge and latch in oxidative stress response. Mol. Cell. Biol. 27, 7511-7521

42 Cino, E. A., Killoran, R. C., Karttunen, M. and Choy, W. Y. (2013) Binding of disordered proteins to a protein hub. Sci. Rep. 3, 2305

43 Cino, E., Fan, J., Yang, D. and Choy, W. Y. (2013) (1)H, (1)(5)N and (1)(3)C backbone resonance assignments of the Kelch domain of mouse Keap1. Biomol. NMR Assign. 7, 149-153

44 Delaglio, F., Grzesiek, S., Vuister, G. W., Zhu, G., Pfeifer, J. and Bax, A. (1995) NMRPipe: a multidimensional spectral processing system based on UNIX pipes. J. Biomol. NMR. 6, 277-293

45 Johnson, B. A. (2004) Using NMRView to visualize and analyze the NMR spectra of macromolecules. Methods Mol. Biol. 278, 313-352

46 Whitmore, L. and Wallace, B. A. (2008) Protein secondary structure analyses from circular dichroism spectroscopy: methods and reference databases. Biopolymers. 89, 392-400 
47 Fukutomi, T., Takagi, K., Mizushima, T., Ohuchi, N. and Yamamoto, M. (2014) Kinetic, thermodynamic, and structural characterizations of the association between Nrf2-DLGex degron and Keap1. Mol. Cell. Biol. 34, 832-846

48 Baird, L., Lleres, D., Swift, S. and Dinkova-Kostova, A. T. (2013) Regulatory flexibility in the Nrf2-mediated stress response is conferred by conformational cycling of the Keap1-Nrf2 protein complex. Proc. Natl. Acad. Sci. U. S. A. 110, 15259-15264 
Table 1. Thermodynamic parameters for the binding of the Neh2 and ProT $\alpha$ peptides to the Kelch domain of human Keap1 at $25^{\circ} \mathrm{C}$

\begin{tabular}{|c|c|c|c|c|c|c|}
\hline $\begin{array}{l}\text { ENGE peptide } \\
\text { of ProT } \alpha\end{array}$ & $n^{*}$ & $\begin{array}{c}K_{\mathrm{a}}^{*} \\
\left(10^{5} \mathrm{M}^{-1}\right)\end{array}$ & $\begin{array}{c}K_{\mathrm{d}}{ }^{*} \\
(\mu \mathrm{M})\end{array}$ & $\begin{array}{c}\Delta \mathbf{H}^{*} \\
(\mathrm{kcal} / \mathrm{mol})\end{array}$ & $\begin{array}{c}\mathrm{T} \Delta \mathrm{S}^{*} \\
\text { (kcal/mol) }\end{array}$ & $\begin{array}{c}\Delta \mathrm{G}^{*} \\
(\mathrm{kcal} / \mathrm{mol})\end{array}$ \\
\hline $\begin{array}{l}\text { Wild-type } \\
\text { Kelch }\end{array}$ & $1.07 \pm 0.004$ & $3.78 \pm 0.15$ & $2.65 \pm 0.11$ & $-17.1 \pm 0.2$ & -9.50 & $-7.60 \pm 0.02$ \\
\hline G350S & $0.92 \pm 0.02$ & $3.33 \pm 0.07$ & $3.00 \pm 0.06$ & $-18.1 \pm 0.6$ & -10.58 & $-7.52 \pm 0.01$ \\
\hline G364C & $0.98 \pm 0.01$ & $0.32 \pm 0.02$ & $31.3 \pm 2.0$ & $-14.17 \pm 0.2$ & -8.03 & $-6.14 \pm 0.04$ \\
\hline R415G & - & - & - & - & - & - \\
\hline A427V & $1.06 \pm 0.003$ & $5.25 \pm 0.1$ & $1.90 \pm 0.04$ & $-22.4 \pm 0.1$ & -14.60 & $-7.80 \pm 0.02$ \\
\hline $\begin{array}{l}\text { ETGE peptide } \\
\text { of Neh2 }\end{array}$ & $n^{*}$ & $\begin{array}{c}K_{\mathrm{a}}^{*} \\
\left(10^{7} M^{-1}\right) \\
\end{array}$ & $\begin{array}{c}K_{\mathrm{d}}{ }^{*} \\
(\mathrm{nM}) \\
\end{array}$ & $\begin{array}{c}\Delta \mathbf{H}^{*} \\
(\mathrm{kcal} / \mathrm{mol})\end{array}$ & $\begin{array}{c}\mathrm{T} \Delta \mathrm{S}^{*} \\
(\mathrm{kcal} / \mathrm{mol})\end{array}$ & $\begin{array}{c}\Delta \mathrm{G}^{*} \\
(\mathrm{kcal} / \mathrm{mol})\end{array}$ \\
\hline $\begin{array}{l}\text { Wild-type } \\
\text { Kelch }\end{array}$ & $1.03 \pm 0.007$ & $3.18 \pm 0.23$ & $31.4 \pm 2.3$ & $-20.4 \pm 0.09$ & -10.17 & $-10.23 \pm 0.04$ \\
\hline G350S & $1.01 \pm 0.005$ & $2.65 \pm 0.3$ & $37.7 \pm 4.3$ & $-18.9 \pm 0.17$ & -8.78 & $-10.12 \pm 0.06$ \\
\hline G364C & $1.07 \pm 0.03$ & $0.29 \pm 0.01$ & $345 \pm 12$ & $-11.53 \pm 0.4$ & -2.72 & $-8.81 \pm 0.02$ \\
\hline R415G & - & - & - & - & - & - \\
\hline A427V & $1.11 \pm 0.002$ & $6.44 \pm 0.67$ & $15.5 \pm 1.6$ & $-19.6 \pm 0.13$ & -8.95 & $-10.65 \pm 0.06$ \\
\hline
\end{tabular}

${ }^{*} \mathrm{n}$ is the stoichiometry, $\mathrm{K}_{\mathrm{a}}$, and $\mathrm{K}_{\mathrm{d}}$ are the association and dissociation constants, respectively. $\Delta \mathrm{G}=\Delta \mathrm{H}-\mathrm{T} \Delta \mathrm{S}=-\mathrm{RT} \ln K_{a}$, where $\mathrm{T}$ is the temperature in Kelvin, $\mathrm{R}$ is the gas constant. $\Delta \mathrm{H}$ and $\Delta \mathrm{S}$ are the enthalpy and entropy changes, respectively. The corresponding ITC isotherms are shown in Figure S2 in supplemental data. Values listed in the table are based on one run, and the data for the duplicate run is provided in the supplementary data (Table S3). 
Table 2. Thermodynamic parameters for the binding of the wild-type and mutational variants of Kelch to the ETGE and DLG sites of full length Neh2 at $5^{\circ}$ C.

\begin{tabular}{|c|c|c|c|c|c|c|c|}
\hline \multicolumn{2}{|c|}{ Protein } & $n$ & $\begin{array}{c}K_{\mathrm{a}} \\
\left(10^{7} \mathbf{M}^{-1}\right)\end{array}$ & $\begin{array}{c}K_{\mathrm{d}} \\
(\mathrm{nM})\end{array}$ & $\underset{(\mathrm{kcal} / \mathrm{mol})}{\Delta \mathbf{H}}$ & $\begin{array}{c}\mathrm{T} \Delta \mathrm{S} \\
(\mathrm{kcal} / \mathrm{mol})\end{array}$ & $\underset{(\mathrm{kcal} / \mathrm{mol})}{\Delta \mathbf{G}^{2}}$ \\
\hline \multirow[t]{2}{*}{ Kelch } & ETGE & $\begin{array}{l}0.90 \pm \\
0.00(3)\end{array}$ & $79.2 \pm 2.07$ & $1.26 \pm 0.03$ & $-20.34 \pm 0.12$ & -9.02 & $-11.32 \pm 0.01$ \\
\hline & DLG & $0.93 \pm 0.02$ & $0.56 \pm \mathbf{0 . 0 1 2}$ & $179 \pm 4$ & $9.04 \pm 0.03$ & 17.62 & $-8.58 \pm 0.01$ \\
\hline \multirow[t]{2}{*}{ G350S } & ETGE & $0.91 \pm 0.01$ & $60.9 \pm 2.99$ & $1.64 \pm 0.08$ & $-20.13 \pm 0.07$ & -8.96 & $-11.17 \pm 0.03$ \\
\hline & DLG & $0.93 \pm 0.01$ & $0.35 \pm 0.014$ & $286 \pm 11$ & $10.61 \pm 0.12$ & 18.93 & $-8.32 \pm 0.02$ \\
\hline \multirow[t]{2}{*}{ G364C } & ETGE & $0.92 \pm 0.01$ & $2.15 \pm 0.18$ & $46.5 \pm 3.9$ & $-15.09 \pm 0.06$ & -5.77 & $-9.32 \pm 0.04$ \\
\hline & DLG & - & - & - & - & - & - \\
\hline \multirow[t]{2}{*}{ R415G } & ETGE & $1.03 \pm 0.01$ & $0.091 \pm 0.008$ & $1099 \pm 97$ & $-13.35 \pm 0.12$ & -5.77 & $-7.58 \pm 0.05$ \\
\hline & DLG & - & - & - & - & - & - \\
\hline \multirow[t]{2}{*}{ A427V } & ETGE & $0.91 \pm 0.01$ & $79.1 \pm 4.9$ & $1.26 \pm 0.08$ & $-20.17 \pm 0.17$ & -8.85 & $-11.32 \pm 0.03$ \\
\hline & DLG & $1.00 \pm 0.03$ & $0.56 \pm 0.021$ & $179 \pm 7$ & $9.44 \pm 0.10$ & 18.02 & $-8.58 \pm 0.02$ \\
\hline
\end{tabular}

The isotherms of wild-type Kelch, G350S and A427V mutants were fit to a two-sitebinding model, assuming that the binding affinities of the ETGE motif are higher than that of the DLG motif. The isotherms of G364C and R415G mutants were fit to a onesite-binding model. $n$ is the stoichiometry, $K_{a}$, and $K_{d}$ are the association and dissociation constants, respectively. $\Delta \mathrm{G}=\Delta \mathrm{H}-\mathrm{T} \Delta \mathrm{S}=-\mathrm{RT} \ln K_{a}$, where $\mathrm{T}$ is the temperature in Kelvin, $\mathrm{R}$ is the gas constant, $\Delta \mathrm{H}$ and $\Delta \mathrm{S}$ are the enthalpy and entropy changes, respectively. Values listed in the table are based on one run, the data for the duplicate run is provided in the Supplementary Data (Table S5). 


\section{FIGURE LEGENDS}

Figure 1. Crystal structure of Kelch with Neh2 and mutation sites located in the Kelch domain of Keap1 (PDB:1U6D [36, 39]). (A) Domain scheme of Keap1 (B) Neh2-ETGE peptide bound to Kelch domain (PDB: 2FLU [36]). (C) Somatic mutations occurring in the binding interface. (D) Mutations found outside the binding interface. (E) Mutations in the conserved double glycine repeats.

Figure 2. Backbone resonance assignments of the Kelch domain of human Keap1 (A) ${ }^{1} \mathrm{H}-{ }^{15} \mathrm{~N}$ HSQC spectrum of ${ }^{2} \mathrm{H} /{ }^{15} \mathrm{~N} /{ }^{13} \mathrm{C}$ labeled Kelch domain of Keap1. (B) Amino acid sequence of the Kelch domain, unassigned residues are colored in red. Amino acids appearing in grey are residual residues from the affinity tag.

Figure 3. ${ }^{1} \mathrm{H}_{\mathrm{N}}$ and ${ }^{15} \mathrm{~N}$ chemical shift changes of the Kelch mutants. Residues with traceable assigned resonances are colored based on composite ${ }^{1} \mathrm{H}_{\mathrm{N}}$ and ${ }^{15} \mathrm{~N}$ chemical shift changes $\left(\Delta \delta=\left[\left(\Delta \delta_{\mathrm{HN}}\right)^{2}+\left(\Delta \delta_{\mathrm{N}} / 5\right)^{2}\right]^{1 / 2}\right.$ in ppm). (A) G350S, (B) G364C, (C) A427V and (D) R415G.

Figure 4. ${ }^{1} \mathrm{H}_{-}{ }^{15} \mathrm{~N}$ HSQC NMR spectra of the wild-type Kelch domain in the absence (black) and presence (red) of five molar equivalents of Neh2-ETGE peptide (A), Neh2DLG peptide (B), and ProT $\alpha$ peptide (C).

Figure 5. Composite ${ }^{1} \mathrm{H}_{\mathrm{N}}$ and ${ }^{15} \mathrm{~N}$ chemical shift perturbation $\left(\Delta \delta=\left[\left(\Delta \delta_{\mathrm{HN}}\right)^{2}+\left(\Delta \delta_{\mathrm{N}} / 5\right)^{2}\right]^{1 / 2}\right)$ analysis of the wild type and the four mutants in the presence of two molar equivalents of ProT $\alpha$ peptide (A, B), two molar equivalents of Neh2-ETGE peptide (C, D), and five molar equivalents of Neh2-DLG peptide (E, F). The boundaries of the propeller blades are defined based on [39]. 
Table S1. Somatic mutations included in this study $\dagger$.

\begin{tabular}{cccc}
\hline Type & $\begin{array}{c}\text { Location in } \\
\text { Kelch }\end{array}$ & Specimen & $\begin{array}{c}\text { Cancer } \\
\text { type }\end{array}$ \\
\hline G364C & Blade II & Cell line & Lung (ADC) \\
R415G & Blade III & Patient & Lung (ADC) \\
R413L & Blade III & Patient & Lung \\
& Blade I & Cell lines and & Lung \\
G350S & Patient & Lung (SCC) \\
A427V & Plade III & Patient & \\
& Blade I & Cell line A549 & Lung \\
G333C & Blade II & Patient & Gall Bladder \\
G379D & Blade III & Patient & Lung (ADC) \\
G430C & Blade IV & Patient & Lung (LCC) \\
G476R &
\end{tabular}

Abbreviations: ADC: Adenocarcinoma, SCC: Small cell carcinoma, LCC: Large cell carcinoma. †Reference: Hayes \& McMahon (2009) Trends Biochem Sci, 34, 176-188 [11]. 

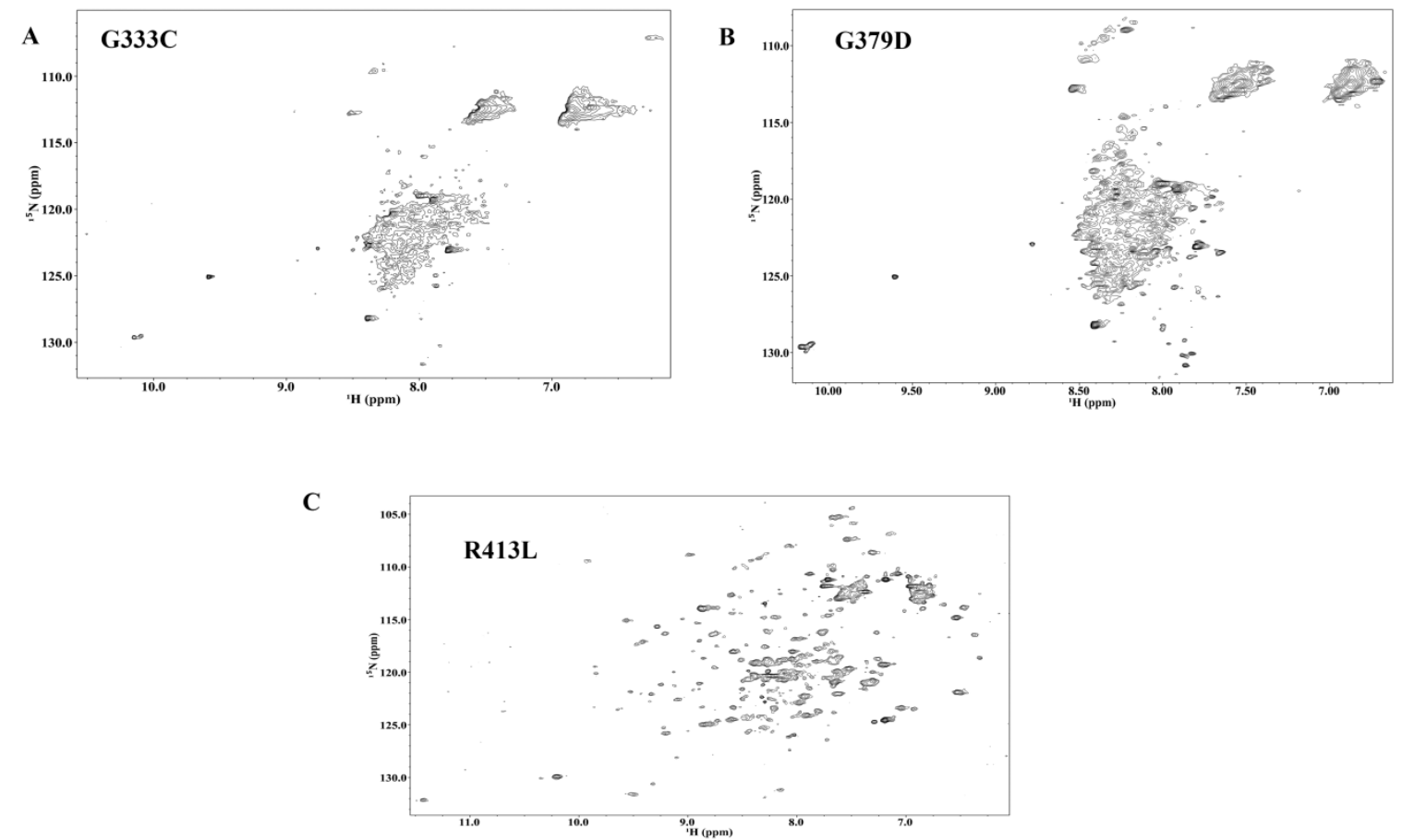

Figure S1. ${ }^{1} \mathrm{H}^{15} \mathrm{~N}$ HSQC NMR spectra of G333C (A), G379D (B), and R413L (C) mutants of the Kelch domain. 


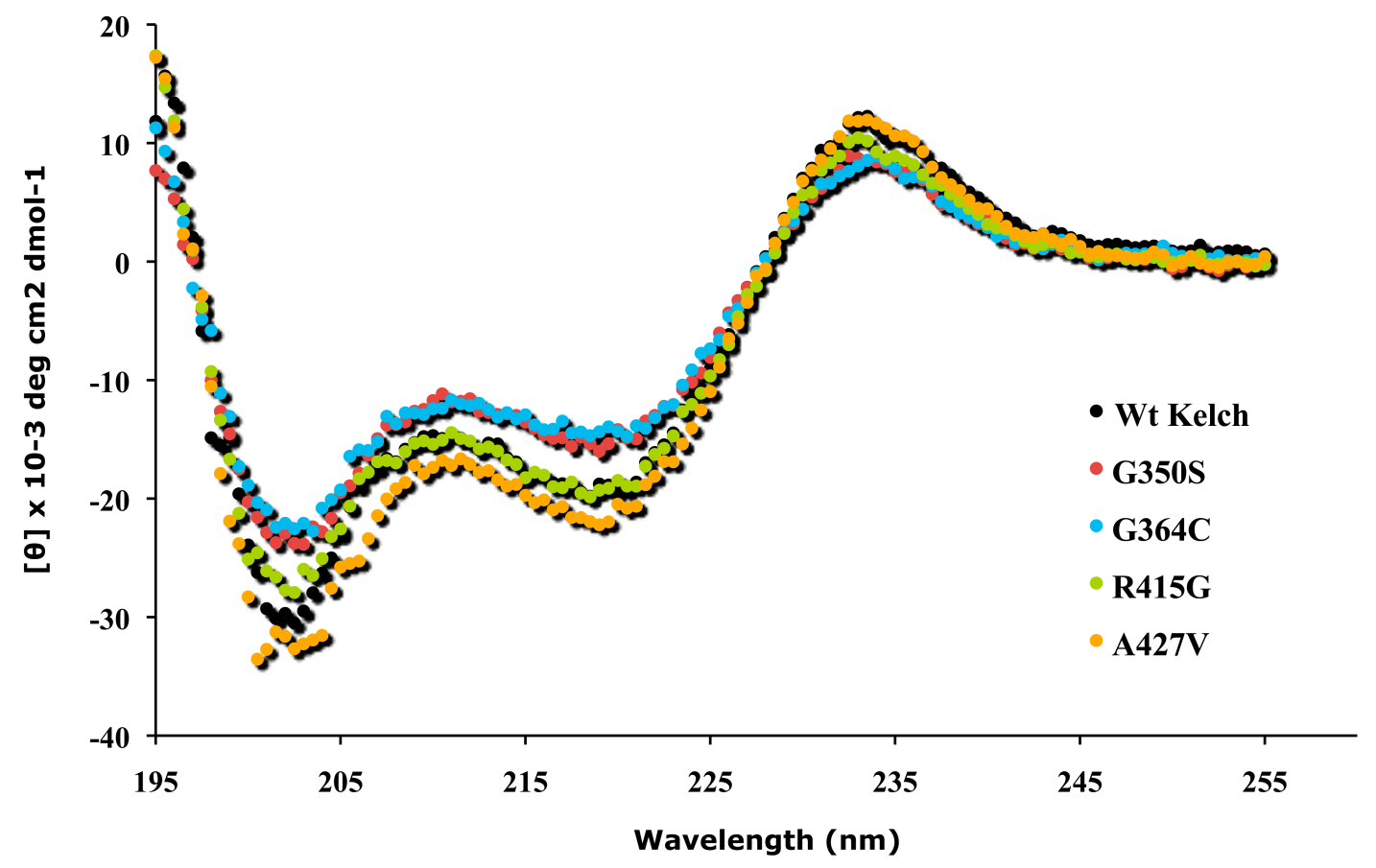

Figure S2. CD spectra of wild-type Kelch (black), G350S (red), G364C (blue), R415G (green), and A427V (orange). 
Table S2. Deconvulated CD data of the WT Kelch and mutational variants

\begin{tabular}{lcccc}
\hline Protein & Helix & $\boldsymbol{\beta}$-sheet & Turn & Unordered \\
\hline WT-Kelch & 0.063 & 0.432 & 0.120 & 0.385 \\
G350S & 0.063 & 0.433 & 0.120 & 0.384 \\
G364C & 0.063 & 0.432 & 0.118 & 0.386 \\
R415G & 0.062 & 0.415 & 0.120 & 0.394 \\
A427V & 0.061 & 0.436 & 0.119 & 0.384 \\
\hline
\end{tabular}



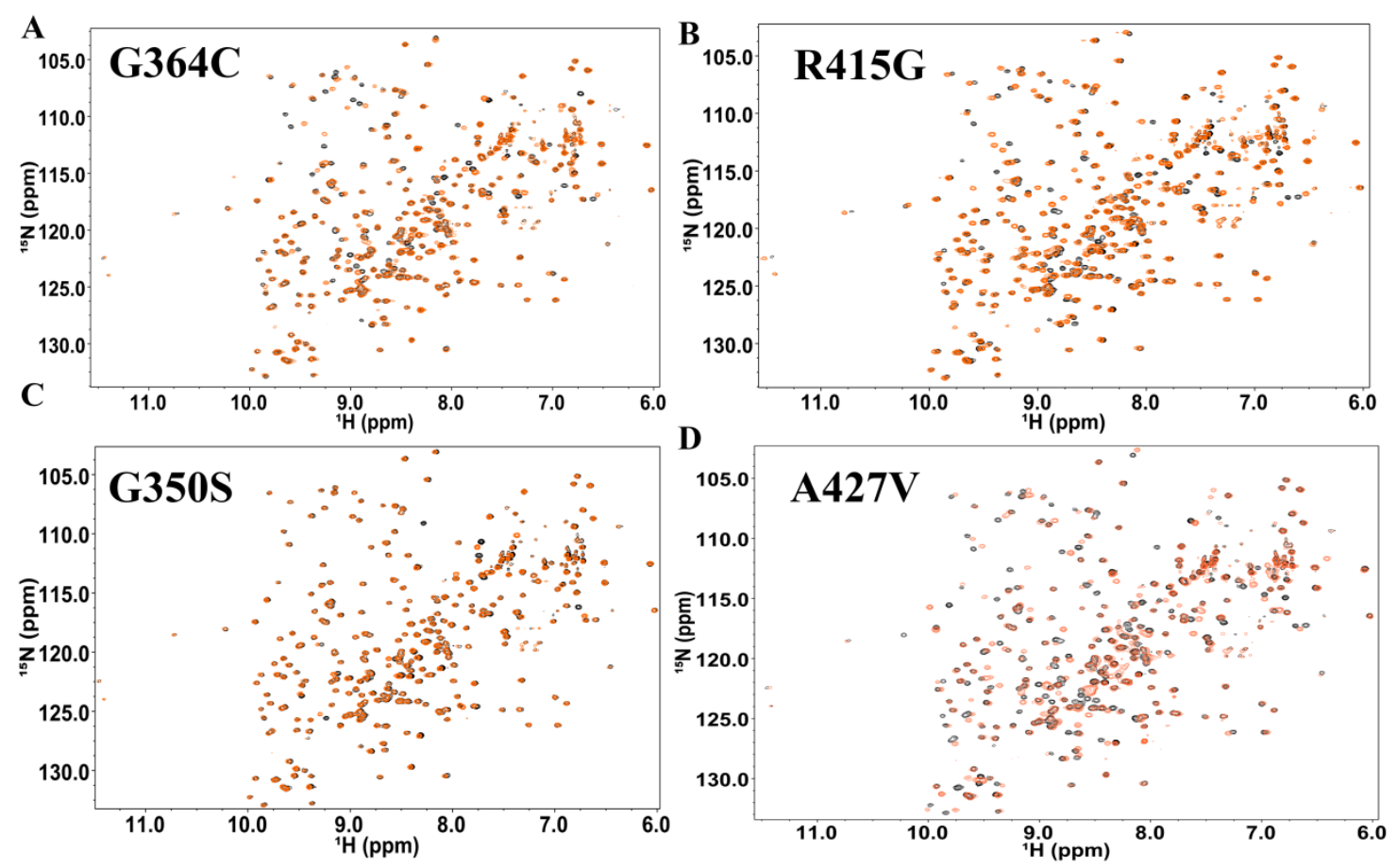

Figure S3. Overlay of the ${ }^{1} \mathrm{H}-{ }^{15} \mathrm{~N}$ TROSY-HSQC NMR spectra of wild-type Kelch (black) and G364C (A), R415G (B), G350S (C), and A427V (D). 

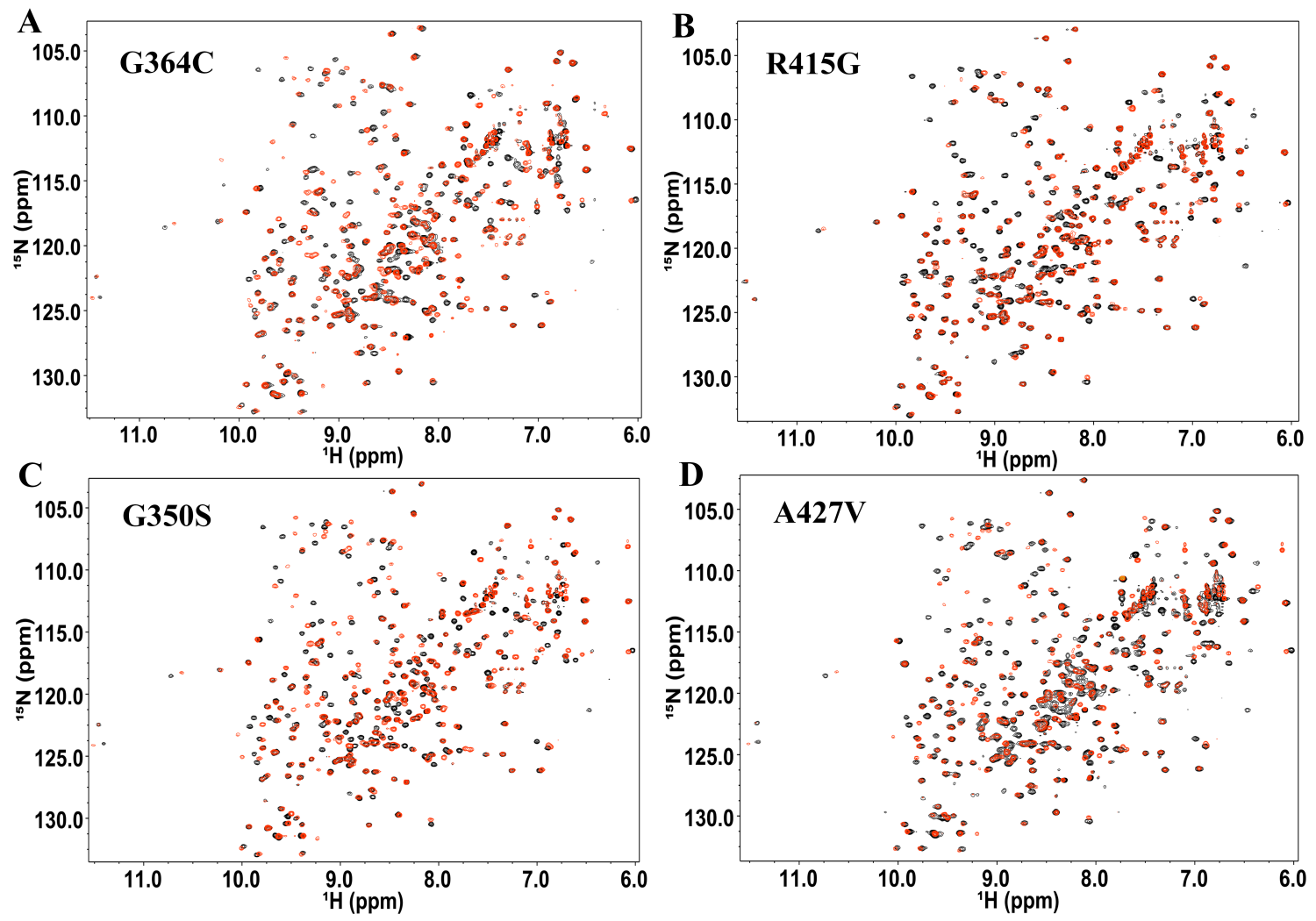

Figure S4. Overlay of the ${ }^{1} \mathrm{H}^{15} \mathrm{~N}$ HSQC NMR spectra of the G364C (A), R415G (B), G350S (C), and A427V (D) Kelch mutations in the absence (black) and presence (orange) of a two molar equivalent of the Neh2-ETGE peptide. 

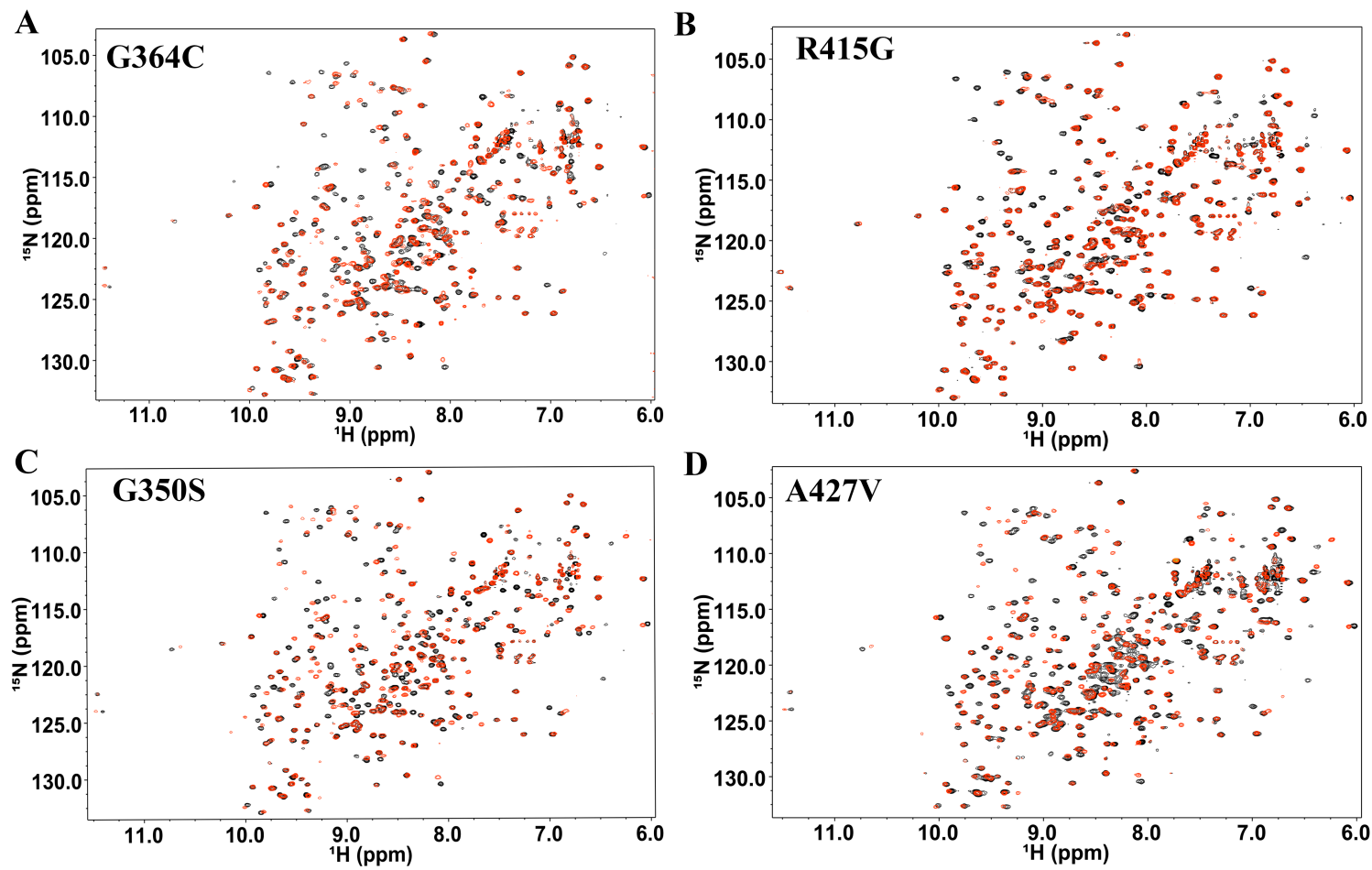

Figure S5. Overlay of the ${ }^{1} \mathrm{H}^{15} \mathrm{~N}$ HSQC NMR spectra of the G364C (A), R415G (B), G350S (C), and A427V (D) Kelch mutations in the absence (black) and presence (orange) of a two molar equivalent of the ProT $\alpha$-ENGE peptide. 

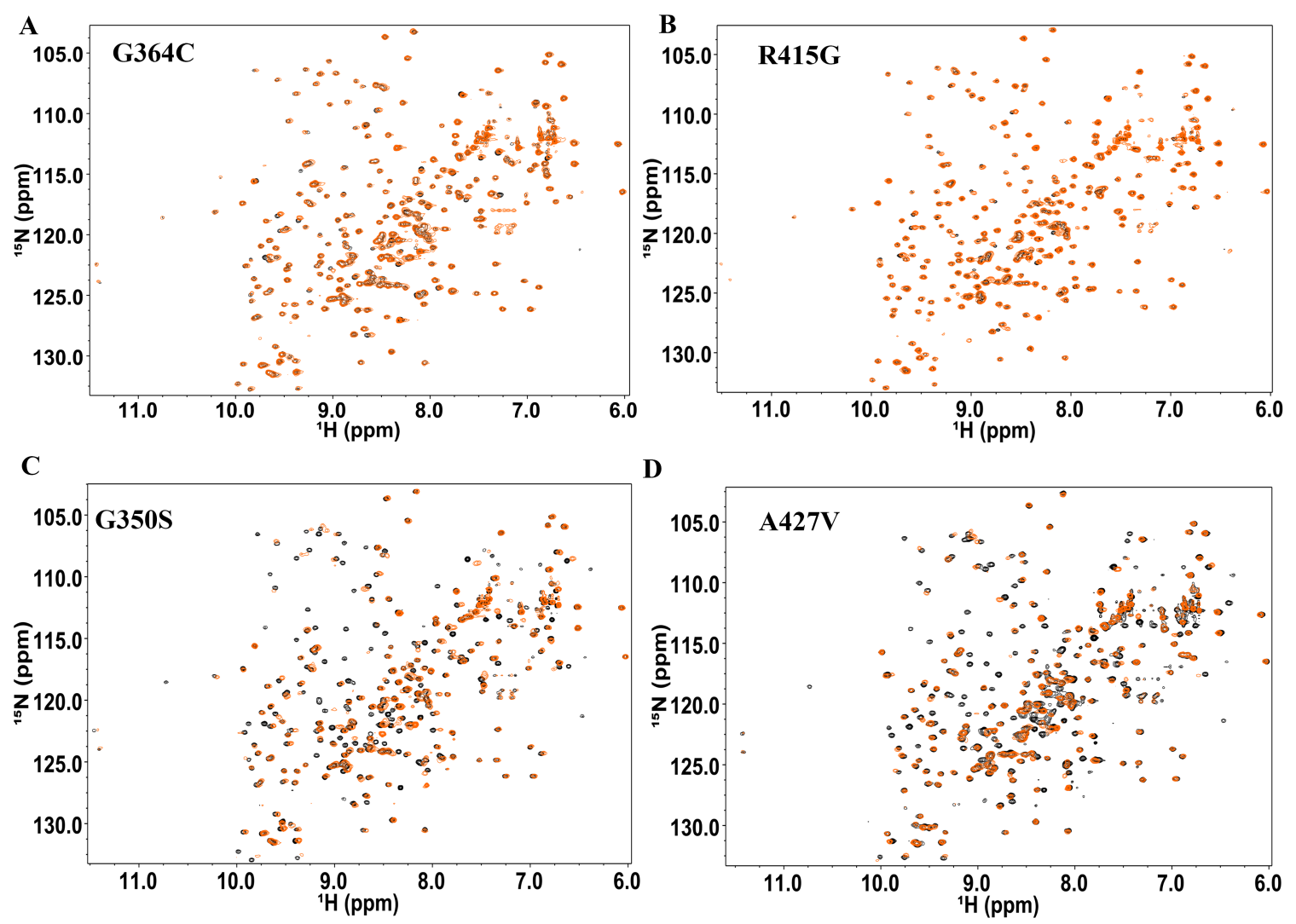

Figure S6. Overlay of the ${ }^{1} \mathrm{H}_{-}{ }^{15} \mathrm{~N}$ HSQC NMR spectra of the Kelch G364C (A), R415G (B), G350S (C), and A427V (D) mutations in the absence (black) and presence (orange) of a five molar equivalent of the Neh2-DLG peptide. 
Table S3. Thermodynamic parameters for the binding of the Neh2 and Prothymosin $\alpha$ (ProT $\alpha$ ) peptides to the human Kelch domain of Keap1 at $25^{\circ} \mathrm{C}$ (duplicate run).

\begin{tabular}{|c|c|c|c|c|c|c|}
\hline $\begin{array}{l}\text { ENGE peptide } \\
\text { of ProT } \alpha\end{array}$ & $n^{*}$ & $\begin{array}{c}K_{\mathrm{a}}^{*} \\
\left(10^{5} \mathrm{M}^{-1}\right)\end{array}$ & $\begin{array}{c}K_{d}{ }^{*} \\
(\mu \mathrm{M})\end{array}$ & $\begin{array}{c}\Delta \mathbf{H}^{*} \\
(\mathrm{kcal} / \mathrm{mol})\end{array}$ & $\begin{array}{c}\mathrm{T} \Delta \mathrm{S}^{*} \\
(\mathrm{kcal} / \mathrm{mol})\end{array}$ & $\begin{array}{c}\Delta \mathrm{G}^{*} \\
(\mathrm{kcal} / \mathrm{mol})\end{array}$ \\
\hline $\begin{array}{l}\text { Wild-type } \\
\text { Kelch }\end{array}$ & $1.03 \pm 0.005$ & $4.01 \pm 0.2$ & $2.49 \pm 0.12$ & $-17.7 \pm 0.12$ & -10.07 & $-7.64 \pm 0.03$ \\
\hline G350S & $0.96 \pm 0.003$ & $2.73 \pm 0.05$ & $3.66 \pm 0.07$ & $-19.2 \pm 0.11$ & -11.79 & $-7.41 \pm 0.01$ \\
\hline G364C & $1.06 \pm 0.2$ & $0.29 \pm 0.01$ & $34.5 \pm 1.2$ & $-15.9 \pm 0.03$ & -9.82 & $-6.08 \pm 0.02$ \\
\hline R415G & - & - & - & - & - & - \\
\hline A427V & $1.04 \pm 0.004$ & $5.24 \pm 0.2$ & $1.91 \pm 0.07$ & $-22.3 \pm 0.15$ & -14.50 & $-7.80 \pm 0.02$ \\
\hline $\begin{array}{l}\text { ETGE peptide } \\
\text { of Neh2 }\end{array}$ & $n^{*}$ & $\begin{array}{c}K_{\mathrm{a}}^{*} \\
\left(10^{7} \mathrm{M}^{-1}\right)\end{array}$ & $\begin{array}{c}K_{d}^{*} \\
(\mathbf{n M})\end{array}$ & $\begin{array}{c}\Delta H^{*} \\
(\mathrm{kcal} / \mathrm{mol})\end{array}$ & $\begin{array}{c}\mathrm{T} \Delta \mathrm{S}^{*} \\
(\mathrm{kcal} / \mathrm{mol})\end{array}$ & $\begin{array}{c}\Delta \mathrm{G}^{*} \\
(\mathrm{kcal} / \mathrm{mol})\end{array}$ \\
\hline $\begin{array}{l}\text { Wild-type } \\
\text { Kelch }\end{array}$ & $1.01 \pm 0.002$ & $3.43 \pm 0.5$ & $29.2 \pm 4.4$ & $-21.0 \pm 0.18$ & -10.73 & $-10.27 \pm 0.09$ \\
\hline G350S & $1.0 \pm 0.003$ & $2.62 \pm 0.3$ & $38.2 \pm 4.3$ & $-19.2 \pm 0.11$ & -9.09 & $-10.11 \pm 0.06$ \\
\hline G364C & $1.08 \pm 0.03$ & $0.4 \pm 0.02$ & $250 \pm 13$ & $-10.63 \pm 0.04$ & -1.63 & $-9.0 \pm 0.03$ \\
\hline R415G & - & - & - & - & - & - \\
\hline A427V & $1.05 \pm 0.003$ & $4.33 \pm 0.5$ & $23.1 \pm 2.7$ & $-19.7 \pm 0.19$ & -9.29 & $-10.41 \pm 0.07$ \\
\hline
\end{tabular}

$*_{n}$ is the stoichiometry, $K_{a}$, and $K_{d}$ are the association and dissociation constants, respectively. $\Delta G=\Delta \mathrm{H}-\mathrm{T} \Delta \mathrm{S}=-R T \ln K_{a}$, where $T$ is the temperature in Kelvin, $R$ is the gas constant. $\Delta H$ and $\Delta S$ are the enthalpy and entropy changes, respectively. Values listed in the table are based on one run, and the data for the duplicate run is provided in Table 1. 

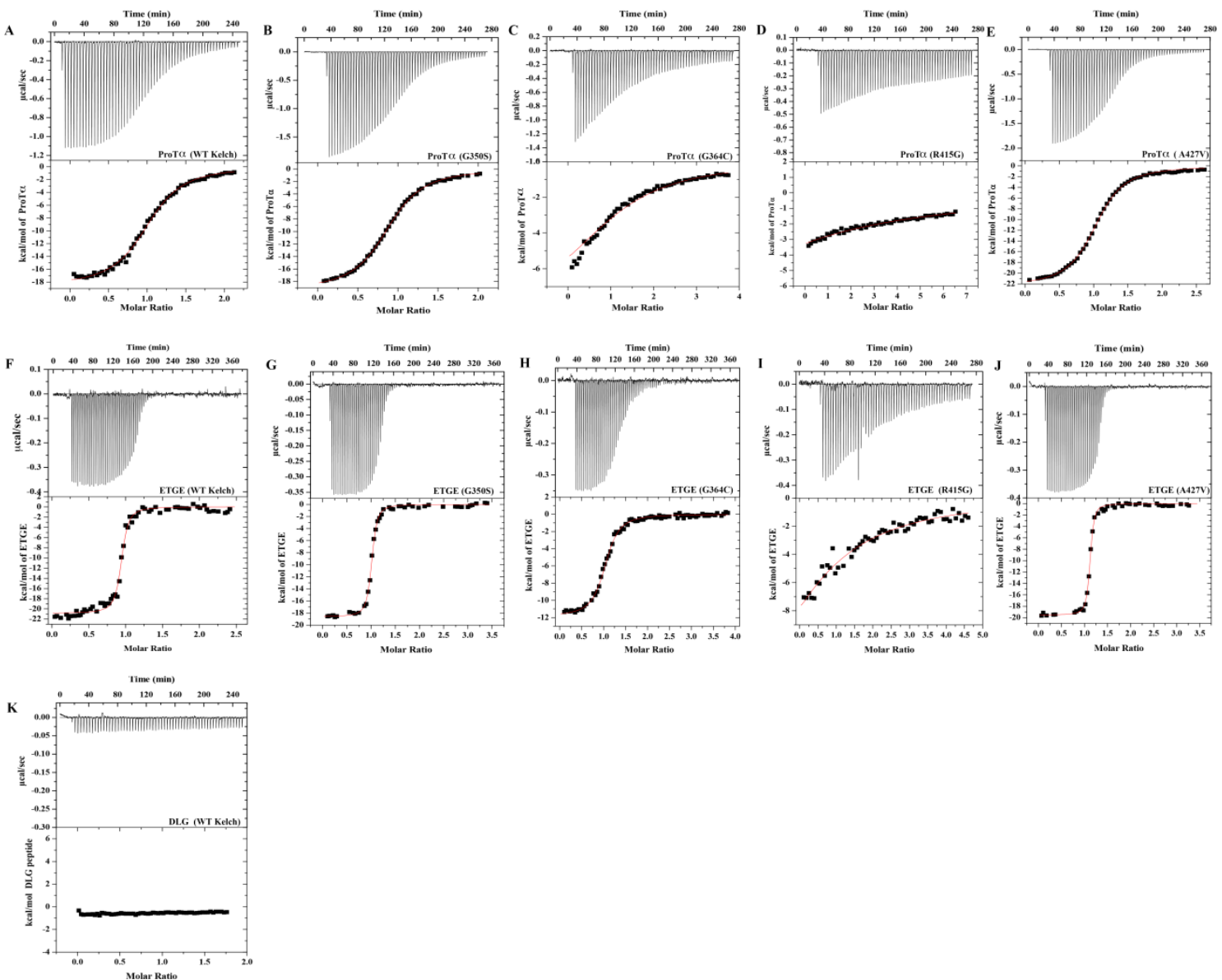

Figure S7. ITC profiles of titrating ProTa-ENGE peptide to wild-type Kelch (A), G350S (B), G364C (C), R415G (D), and A427V (E), respectively; (F-J) show the ITC profiles of titrating Neh2-ETGE peptide to wild-type Kelch (F), G350S (G), G364C (H), R415G (I), and A427V (J), respectively; (K) ITC profile of titrating Neh2-DLG peptide to wild-type Kelch. 


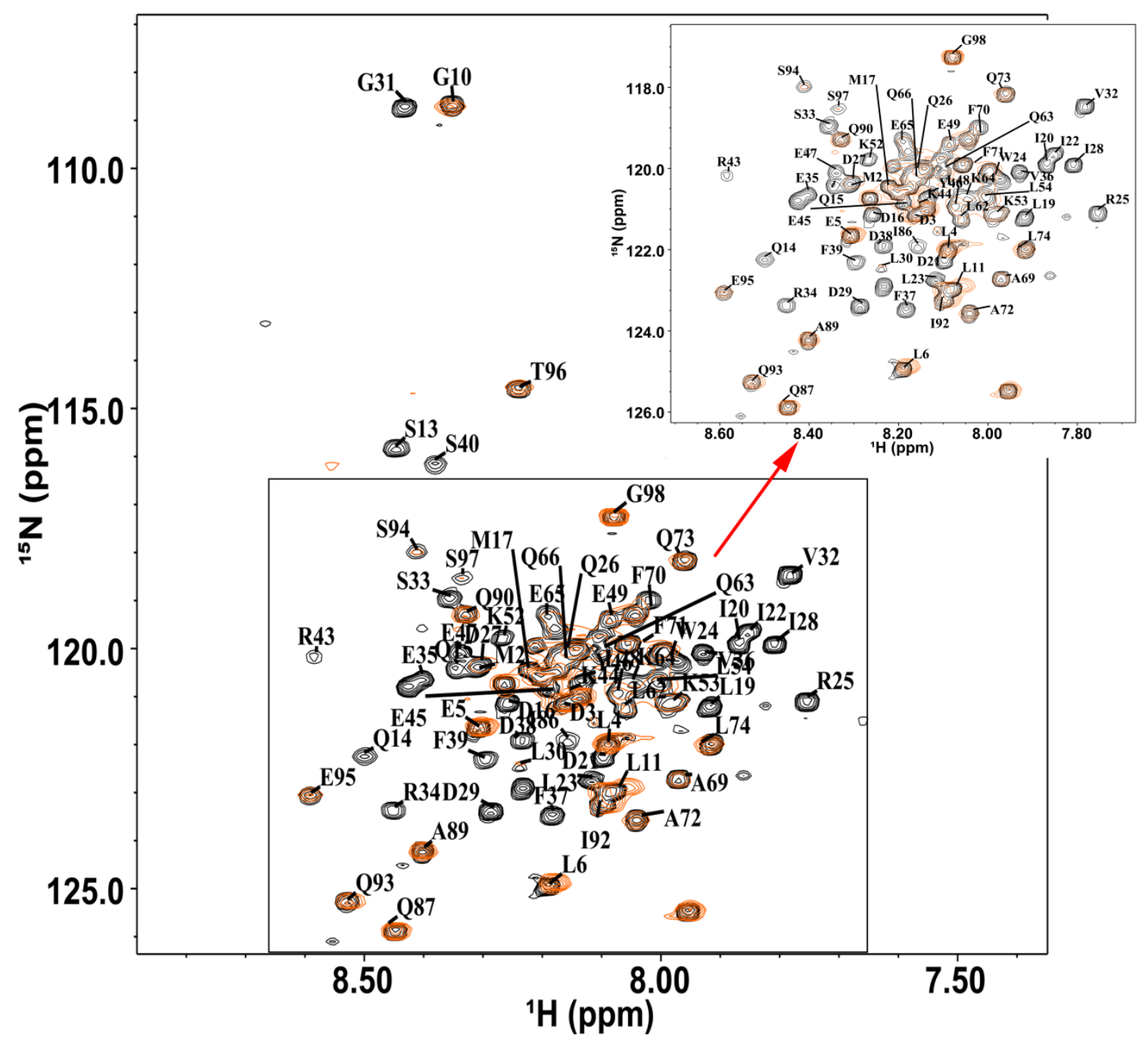

Figure S8. ${ }^{1} \mathrm{H}-{ }^{15} \mathrm{~N}$ HSQC of Neh2 $\triangle \mathrm{ETGE}$ in the free (black) and Kelch bound (orange) state. Many residues in the N-terminal region (L6, L11, S13, Q14, L23, R25 Q14, D16, I20, W24, I28, D29, L30, G31, V32, S33, R34, F37, F39 and S40) of Neh2 undergo substantial perturbations suggesting that these flanking residues contribute to the DLG motif interaction with the Kelch domain. 

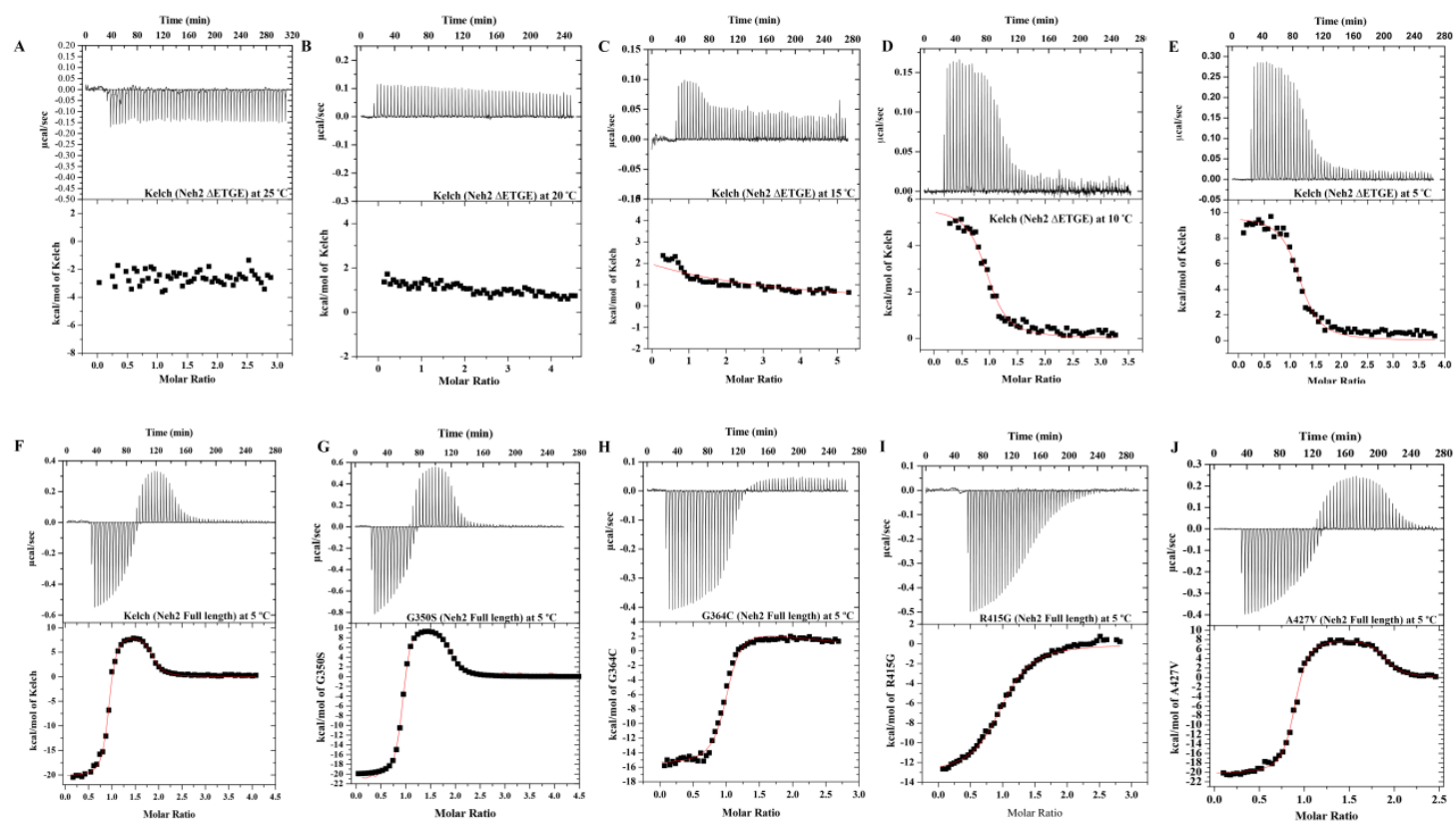

Figure S9. ITC profiles of titrating wild-type Kelch to Neh2 $\triangle$ ETGE at $25{ }^{\circ} \mathrm{C}(\mathrm{A}), 20^{\circ} \mathrm{C}$ (B), $15{ }^{\circ} \mathrm{C}(\mathrm{C}), 10{ }^{\circ} \mathrm{C}(\mathrm{D})$, and $5{ }^{\circ} \mathrm{C}(\mathrm{E})$, respectively. (F-J) shows the ITC profiles of titrating wild-type Kelch (F), G350S (G), G464C (H), R415G (I), and A427V (J), respectively, to full-length $\mathrm{Neh} 2$ at $5^{\circ} \mathrm{C}$. 
Table S4. Thermodynamic parameters for the binding of the Kelch domain of Keap1 to Neh2 $\Delta$ ETGE at different temperatures

\begin{tabular}{|c|c|c|c|c|c|c|}
\hline $\operatorname{Temp}\left({ }^{\circ} \mathrm{C}\right)$ & $n^{*}$ & $\begin{array}{c}K_{\mathrm{a}}{ }^{*} \\
\left(10^{6} \mathrm{M}^{-1}\right)\end{array}$ & $\begin{array}{c}K_{\mathrm{d}}{ }^{*} \\
(\mathrm{nM})\end{array}$ & $\begin{array}{c}\Delta \mathbf{H}^{*} \\
(\mathrm{kcal} / \mathrm{mol})\end{array}$ & $\begin{array}{c}\mathrm{T} \Delta \mathrm{S}^{*} \\
\text { (kcal/mol) }\end{array}$ & $\begin{array}{c}\Delta \mathrm{G}^{*} \\
(\mathrm{kcal} / \mathrm{mol})\end{array}$ \\
\hline \multicolumn{7}{|l|}{ Titration set 1} \\
\hline 5 & $0.90 \pm 0.03$ & $3.03 \pm 0.14$ & $330 \pm 15$ & $8.13 \pm 0.11$ & 16.37 & $-8.24 \pm 0.03$ \\
\hline 10 & $1.02 \pm 0.17$ & $2.85 \pm 0.13$ & $351 \pm 16$ & $5.80 \pm 0.06$ & 14.16 & $-8.36 \pm 0.03$ \\
\hline \multicolumn{7}{|l|}{ Titration set 2} \\
\hline 5 & $1.10 \pm 0.02$ & $3.13 \pm 0.18$ & $319 \pm 18$ & $8.70 \pm 0.15$ & 16.96 & $-8.26 \pm 0.03$ \\
\hline 10 & $0.97 \pm 0.02$ & $2.66 \pm 0.11$ & $376 \pm 16$ & $5.64 \pm 0.04$ & 13.96 & $-8.32 \pm 0.02$ \\
\hline
\end{tabular}

${ }^{*} n$ is the stoichiometry, $K_{a}$, and $K_{d}$ are the association and dissociation constants, respectively. $\Delta G=\Delta \mathrm{H}-\mathrm{T} \Delta \mathrm{S}=-R T \ln K_{a}$, where $T$ is the temperature in Kelvin, $R$ is the gas constant. $\Delta H$ and $\Delta S$ are the enthalpy and entropy changes, respectively. 
Table S5. Thermodynamic parameters for the binding of the Kelch and Kelch mutational variants to the ETGE and DLG sites of full length Neh2 at $5^{\circ} \mathrm{C}$ (duplicate runs).

\begin{tabular}{|c|c|c|c|c|c|c|c|}
\hline Protein & & $n$ & $\begin{array}{c}K_{\mathrm{a}} \\
\left(10^{7} \mathrm{M}^{-1}\right)\end{array}$ & $\begin{array}{c}K_{\mathrm{d}} \\
(\mathbf{n M})\end{array}$ & $\begin{array}{c}\Delta \mathbf{H} \\
(\mathrm{kcal} / \mathrm{mol})\end{array}$ & $\begin{array}{c}\mathrm{T} \Delta \mathrm{S} \\
(\mathrm{kcal} / \mathrm{mol})\end{array}$ & $\begin{array}{c}\Delta G \\
(\mathrm{kcal} / \mathrm{mol})\end{array}$ \\
\hline \multirow[t]{2}{*}{ Kelch } & ETGE & $1.06 \pm 0.04$ & $73.3 \pm 1.84$ & $1.36 \pm 0.03$ & $-19.02 \pm 0.08$ & -7.74 & $-11.28 \pm 0.01$ \\
\hline & DLG & $1.12 \pm 0.02$ & $0.46 \pm 0.010$ & $217 \pm 5$ & $8.46 \pm 0.10$ & 16.93 & $-8.47 \pm 0.01$ \\
\hline \multirow[t]{2}{*}{ G350S } & ETGE & $1.07 \pm 0.01$ & $51.8 \pm 1.79$ & $1.93 \pm 0.07$ & $-19.83 \pm 0.13$ & -8.74 & $-11.08 \pm 0.02$ \\
\hline & DLG & $1.1 \pm 0.03$ & $0.36 \pm 0.011$ & $278 \pm 8$ & $9.52 \pm 0.09$ & 17.86 & $-8.34 \pm 0.02$ \\
\hline \multirow[t]{2}{*}{ G364C } & ETGE & $0.92 \pm 0.01$ & $2.34 \pm 0.12$ & $42.7 \pm 2.2$ & $-15.0 \pm 0.12$ & -5.63 & $-9.37 \pm 0.03$ \\
\hline & DLG & - & - & - & - & - & - \\
\hline \multirow[t]{2}{*}{ R415G } & ETGE & $1.0 \pm 0.01$ & $0.089 \pm 0.008$ & $1124 \pm 101$ & $-13.40 \pm 0.05$ & -5.83 & $-7.57 \pm 0.05$ \\
\hline & DLG & - & - & - & - & - & - \\
\hline \multirow[t]{2}{*}{ A427V } & ETGE & $\begin{array}{c}0.91 \pm \\
0.00(3)\end{array}$ & $68.4 \pm 1.95$ & $1.46 \pm 0.04$ & $-20.69 \pm 0.14$ & -9.45 & $-11.24 \pm 0.02$ \\
\hline & DLG & $1.00 \pm 0.01$ & $0.53 \pm 0.014$ & $189 \pm 5$ & $9.52 \pm 0.02$ & 18.07 & $-8.55 \pm 0.01$ \\
\hline
\end{tabular}

The isotherms of wild-type Kelch, G350S and A427V mutants were fit to a two-sitebinding model, assuming that the binding affinities of the ETGE site are higher than that of the DLG site. The isotherms of G364C and R415G mutants were fit to a one-sitebinding model. $n$ is the stoichiometry, $K_{a}$, and $K_{d}$ are the association and dissociation constants, respectively. $\Delta \mathrm{G}=\Delta \mathrm{H}-\mathrm{T} \Delta \mathrm{S}=-\mathrm{RT} \ln K_{a}$, where $\mathrm{T}$ is the temperature in Kelvin, $\mathrm{R}$ is the gas constant, $\Delta \mathrm{H}$ and $\Delta \mathrm{S}$ are the enthalpy and entropy changes, respectively. 
A

$\left.\begin{array}{r}0.5 \\ 0.45\end{array}\right]$ Prothymosin alpha

$0.4-\mathrm{A} 427 \mathrm{~V}$

$0.35-G 350 \mathrm{~S}$

0.3 - Kelch

$\hat{2}_{0.25}$

0.2 .

0.15

0.05

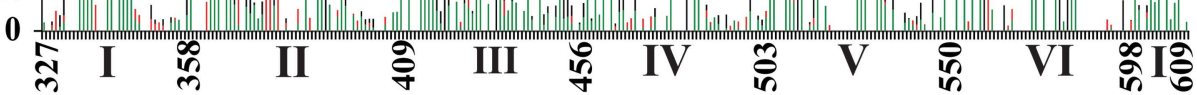

C

$\left.\begin{array}{r}0.5 \\ 0.45\end{array}\right]$ Neh2_ETGE

$0.35-G 350 S$

छ 0.3 - Kelch

气̂. 0.25

io 0.15

0.15

0.05

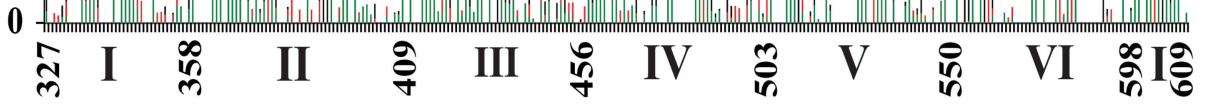

E

$0.45]$ Neh2_DLG

$0.4-\mathrm{A427} \mathrm{V}$

$0.35-G 350 \mathrm{~S}$

छ 0.3 - Kelch

0.25

$\underbrace{2} 0.1$

0.15
0.1
0.05

0.05

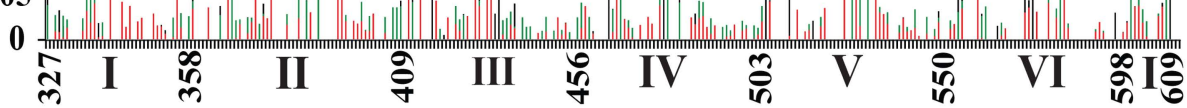

Residue/Blade
B

0.45 Prothymosin alpha

$0.4-G 364 C$

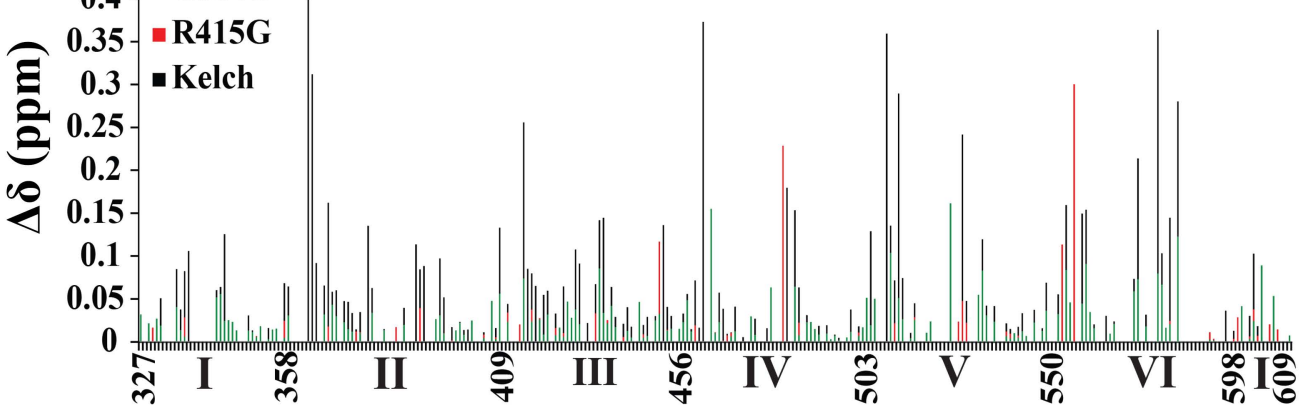

D

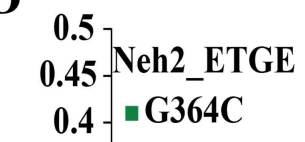

$0.35-\mathrm{R} 415 \mathrm{G}$

ह 0.3 - - Kelch

을 0.25

\& 0.15

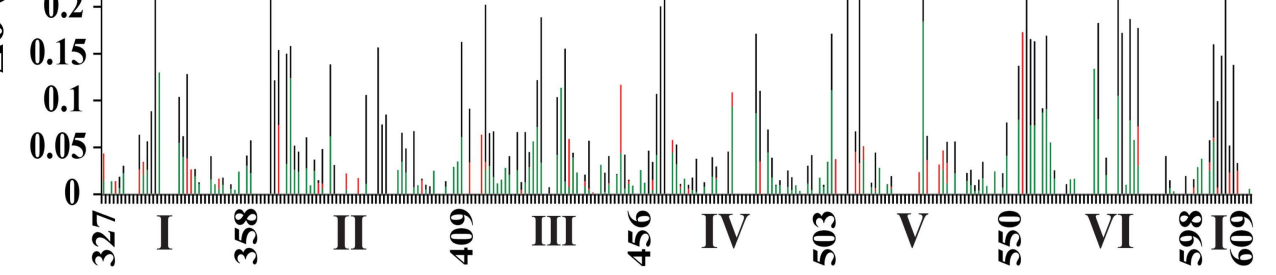

F

$$
\left.\begin{array}{r}
0.5 \\
0.45 \\
0.4
\end{array}\right]=\text { G364C }
$$

20.35- R415G

0.3 - Kelch

을 0.25

0.2

0.15

0.1
0.05

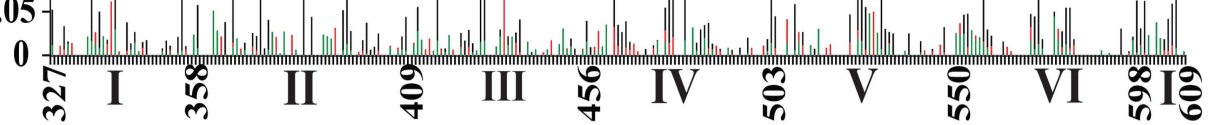

\section{Residue/Blade}




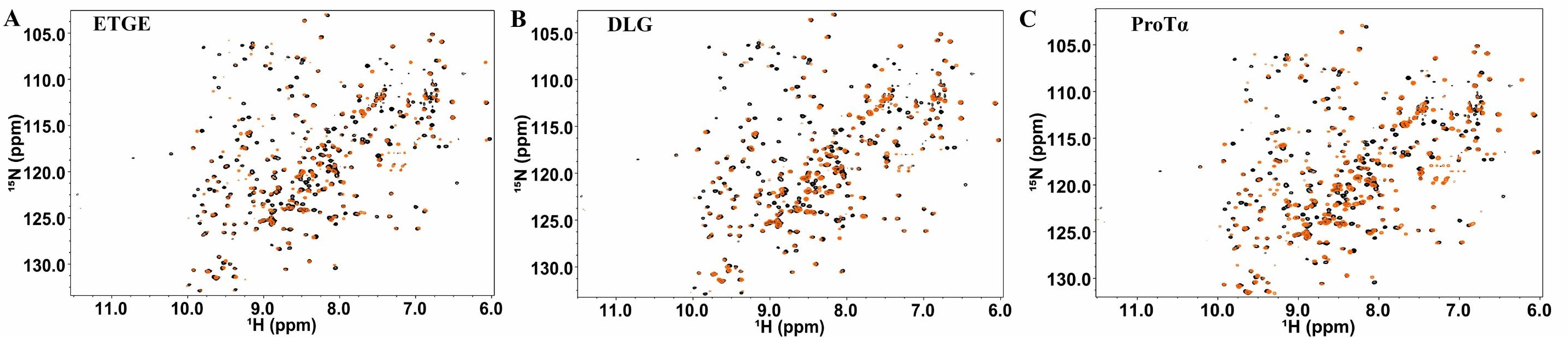




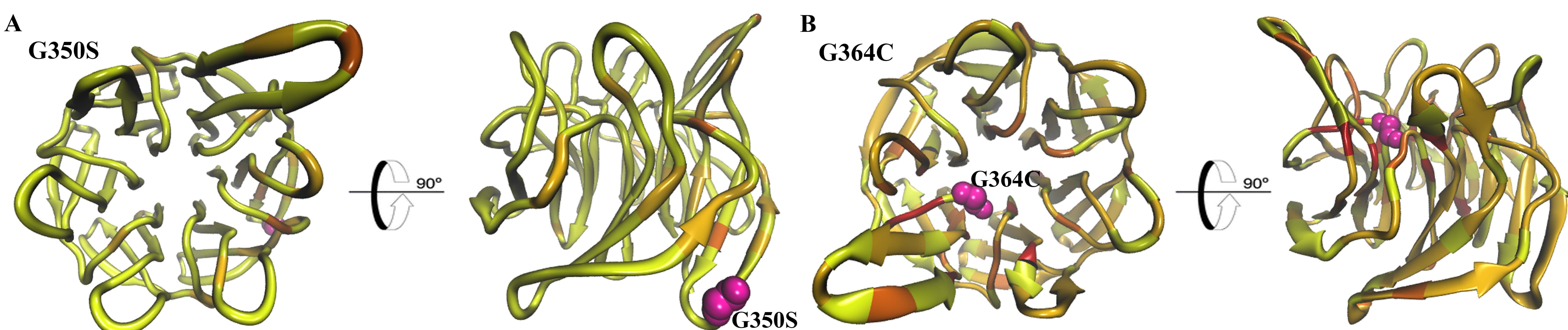

$$
\begin{aligned}
& \text { - }
\end{aligned}
$$




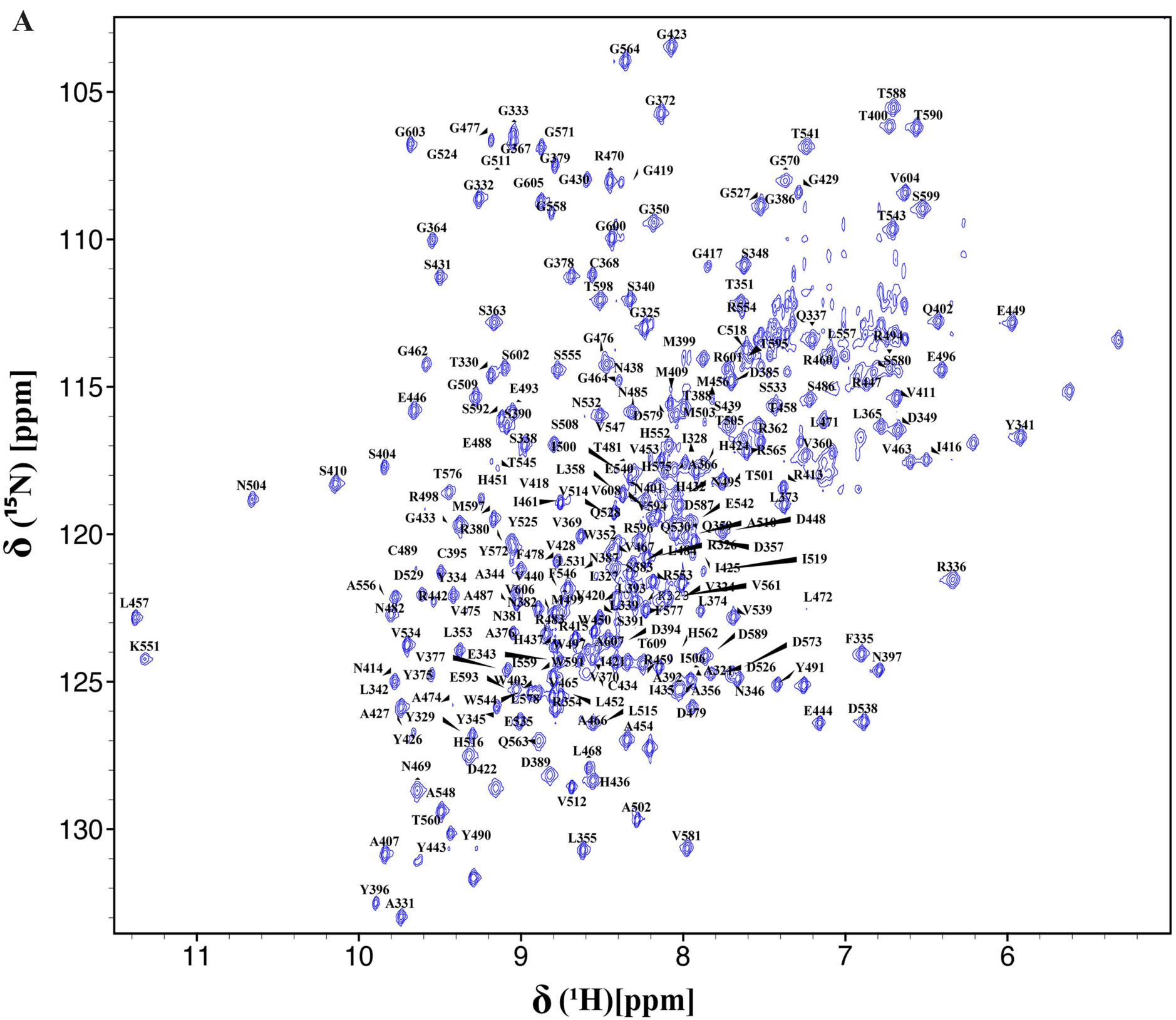

B GSHMAPKVGR LIYTAGGYFR QSLSYLEAYN PSDGTWLRLA DLQVPRSGLA 366 GCVVGGLLYA VGGRNNSPDG NTDSSALDCY NPMTNQWSPC APMSVPRNRI 416 GVGVIDGHIY AVGGSHGCIH HNSVERYEPE RDEWHLVAPM LTRRIGVGVA 466 VLNRLLYAVG GFDGTNRLNS AECYYPERNE WRMITAMNTI RSGAGVCVLH 516 NCIYAAGGYD GQDQLNSVER YDVETETWTF VAPMKHRRSA LGITVHQGRI 566 YVLGGYDGHT FLDSVECYDP DTDTWSEVTR MTSGRSGVGV AVT 
A

Kelch

\begin{tabular}{lllll|l|l|l|l|l|l} 
NTR & BTB & IVR & & & I & II & III & IV & V & VI CTR \\
\hline
\end{tabular}

B

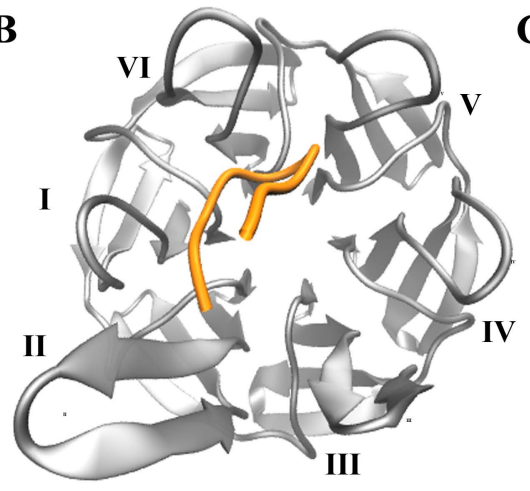

C

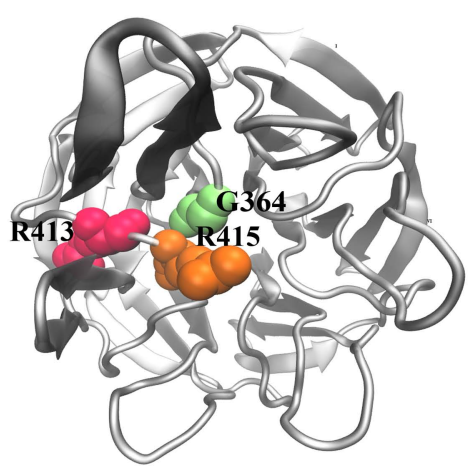

D

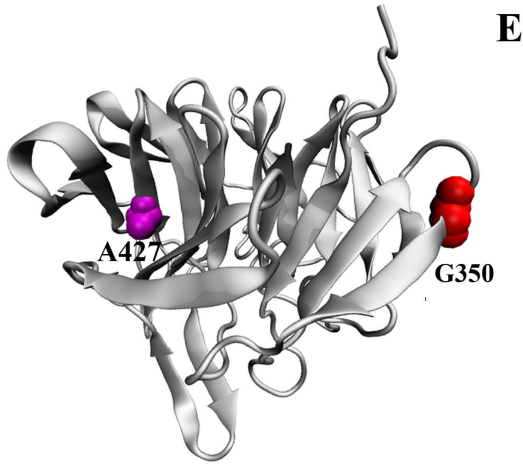

E

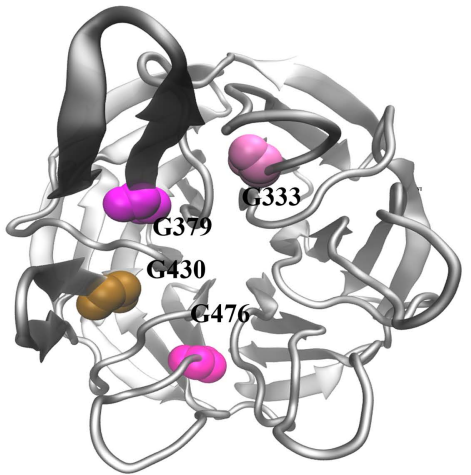

